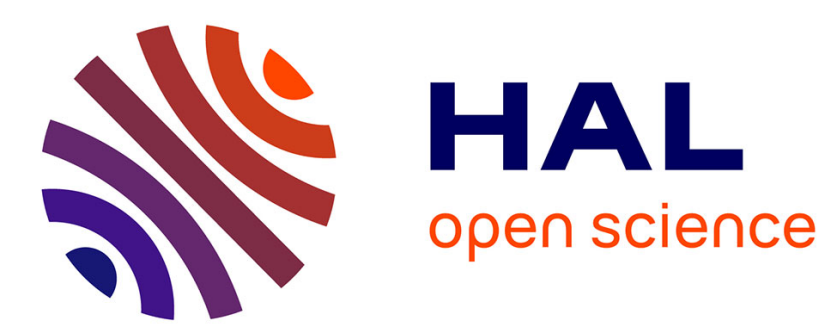

\title{
Topology-induced dynamics in a network of synthetic oscillators with piecewise affine approximation
}

Elena Firippi, Madalena Chaves

\section{To cite this version:}

Elena Firippi, Madalena Chaves. Topology-induced dynamics in a network of synthetic oscillators with piecewise affine approximation. Chaos: An Interdisciplinary Journal of Nonlinear Science, 2020, 30 (11), pp.113128. 10.1063/5.0020670 . hal-03105513

\section{HAL Id: hal-03105513 \\ https://hal.inria.fr/hal-03105513}

Submitted on 11 Jan 2021

HAL is a multi-disciplinary open access archive for the deposit and dissemination of scientific research documents, whether they are published or not. The documents may come from teaching and research institutions in France or abroad, or from public or private research centers.
L'archive ouverte pluridisciplinaire HAL, est destinée au dépôt et à la diffusion de documents scientifiques de niveau recherche, publiés ou non, émanant des établissements d'enseignement et de recherche français ou étrangers, des laboratoires publics ou privés. 


\title{
Topology-induced dynamics in a network of synthetic oscillators with piecewise affine approximation
}

\author{
E. Firippi ${ }^{1, \text { a) }}$ and M. Chaves ${ }^{1, b)}$ \\ Université Côte d'Azur, Inria, INRA, CNRS, Sorbonne Université, Biocore team, Sophia Antipolis, \\ France
}

(Dated: July 3, 2020)

In synthetic biology approaches, minimal systems are used to reproduce complex molecular mechanisms that appear in the core functioning of multi-cellular organisms. In this paper, we study a piecewise affine model of a synthetic twogene oscillator and prove existence and stability of a periodic solution for all parameters in a given region. Motivated by the synchronization of circadian clocks in a cluster of cells, we next consider a network of $N$ identical oscillators under diffusive coupling, to investigate the effect of the topology of interactions in the network's dynamics. Our results show that both all-to-all and one-to-all coupling topologies may introduce new stable steady states, in addition to the expected periodic orbit. Both topologies admit an upper bound on the coupling parameter that prevents the generation of new steady states. However, this upper bound is independent of the number of oscillators in the network and less conservative for the one-to-all-topology.

\begin{abstract}
Intercellular communication is a fundamental part of the development and regulation of living systems. Motivated by the synchronization of biological rhythms in a group of cells, and to gain insight into the effects of network architecture on the system's behaviour, we first analyze a synthetic two-gene oscillator and then study a network of identical oscillators interconnected according to three different topologies. Each two-gene oscillator is modeled in the piecewise affine formalism, and they are connected by diffusive coupling. Using this formalism, we characterize and compare the effect of three different network architectures on the system's dynamics and synchronization properties.
\end{abstract}

\section{INTRODUCTION}

A deeper understanding of cellular complex processes is due to recent research in synthetic biology, which involves the design and implementation of small genetic circuits to mimic the function of more complex biochemical mechanisms. Analysis of these smaller circuits thus contributes to elucidate the origins of complex biological behaviour such as the bacteriophage lambda switch (Gardner, Cantor, and Collins, 2000) or biological rhythms and oscillations (see the repressilator by Elowitz and Leibler (2000) and a review of synthetic oscillators by Purcell et al. (2010)).

New research in synthetic biology is heading towards the use of basic modules (such as a toggle switch or a repressilator) as functional units to (re-)create more complex systems, followed by the control and regulation of these systems (del Vecchio, Dy, and Qian, 2016). In this context, the mathematical analysis of the coupled synthetic modules is essential to predict the behaviour of the new system, investigate the pa-

\footnotetext{
a)Electronic mail: eleni.firippi@inria.fr

b)Electronic mail: madalena.chaves@inria.fr
}

rameter conditions leading to desired behaviour, and generally help experimental design. Examples of such studies include the coupling of different circuits to increase the dynamical range of the system (Perez-Carrasco et al., 2018), form new spatial patterns (Barbier, Perez-Carrasco, and Schaerli, 2020) or the regulation of a bacterial consortium (Fiore et al., 2017).

In this paper, we will consider the coupling of $N$ identical oscillatory circuits, to study some questions relevant in biological rhythms (Hafner, Koeppl, and Gonze, 2012), including the synchronization of the individual circuits or the effect of the topology of interactions and their strength in the robustness of periodic oscillations.

A minimal two gene oscillator that was first proposed by Smolen, Baxter, and Byrne (1998) and later implemented by Stricker et al. (2008), consists of two transcriptional factors competing with each other in a negative feedback loop, together with two self-regulatory loops, one positive and one negative. In previous work, we suggested an improved design for this two gene oscillator by showing that eliminating the self-regulatory loop of the repressor substantially increases the parameter region that admits oscillatory regimes (Firippi and Chaves, 2019). To further analyze this improved circuit, we now write the continuous system in the piecewise affine model framework first introduced by Glass and Kauffman (1973).

This formalism is based on the approximation of sigmoidaltype functions by step functions, and the phase space of the system is typically partitioned into rectangular regular domains, in which the system is affine and solutions can be explicitly computed (Casey, Jong, and Gouzé, 2006). However, in our approximation of the two-gene oscillator, the regular domains are not rectangular but instead defined in terms of a quadratic expression relating the two coordinates. The solutions in each domain can still be explicitly computed, but the concatenation of the different pieces at the boundary between domains, has to be analyzed in a new way. In this new framework, we will compute the Poincaré map of the twodimensional system to prove the existence and uniqueness of a periodic orbit, and will also characterize the corresponding 
parameter region.

As an application of our two-gene oscillator analysis, we then study a network of $N$ identical piecewise affine twogene oscillators, linked by diffusion of one of the variables. There are still few studies of coupled identical oscillators in a piecewise affine formalism: for instance, Edwards and Gill (2003) prove synchronization under a weak coupling, and Nicks, Chambon, and Coombes (2018) study clusters of synchronized elements, with applications to neuron networks.

Our goal is to investigate the effect of the topology of interactions in the network's dynamics, so we consider three different topologies: one-to-all, all-to-all, and random connections. The main result is that all these topologies generate new "coupling" stable steady states, in addition to the periodic orbit, thus possibly contributing to a loss of synchronization and/or a loss of oscillatory behaviour in the coupled network. The generation of new steady states in the coupled system was also observed in Chaves, Scardovi, and Firippi (2019), for a network of coupled positive circuits. The number of new steady states depends on the number $N$ of oscillators in the network and can be exactly computed for both the one-to-all and all-to-all topologies.

For all topologies, we use both numerical simulations and theoretical analysis to study the effect of the coupling parameter, the basins of attraction of the original periodic orbit, and other properties, to establish conditions that guarantee cell synchronization.

\section{A TWO-GENE SYNTHETIC OSCILLATOR}

The original model introduced by Smolen, Baxter, and Byrne (1998) describes a negative feedback circuit of two components, the transcription factors $T F-A$ and $T F-R$ : TF-A is a transcriptional activator that can bind to responsive elements DNA sequences; while TF-R is a protein that represses transcription by competing with TF-A for binding to the responsive elements. TF-A is an activator both for TF-R and itself and, likewise, TF-R is a repressor for both TF-A and itself, leading to a negative feedback loop with a self-activating activator and a self-repressing repressor. For simplicity, $T F-A$ and $T F-R$ are denoted by $A$ and $R$ respectively.

In a previous study (Firippi and Chaves, 2019), through bifurcation analysis, we have found that removing the selfrepressing loop significantly increases the parameter range where oscillations are observed. This leads to the circuit shown in Fig. 1 and described by the following equations:

$$
\begin{aligned}
& \frac{d A}{d t}=V_{A} \frac{A^{2}}{A^{2}+\theta_{0}\left(1+R / \theta_{2}\right)}-\gamma_{A} A+r_{\text {bas }} \\
& \frac{d R}{d t}=V_{R} \frac{A^{2}}{A^{2}+\theta_{1}}-\gamma_{R} R
\end{aligned}
$$

The parameters $V_{A}, V_{R}>0$ express the synthesis rate of the model components and are measured in concentration. min $^{-1}$. The parameters $\gamma_{A}, \gamma_{R}>0$ represent the degradation rates and they are measured in $\mathrm{min}^{-1}$. The concentration and activity thresholds are described by parameters $\theta_{0}, \theta_{1}, \theta_{2}>0$ that also have concentration units.

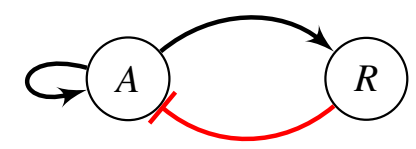

Figure 1. The improved design of the Smolen oscillator, proposed by Firippi and Chaves (2019).

\section{A. Piecewise affine formalism}

The piecewise affine (PWA) systems studied first by Glass and Kauffman (1973) facilitate the system further study and analysis. This formalism is based on the approximation of the Hill functions that express the activation and inhibition (or repression), by increasing and decreasing step functions.

A synopsis of the PWA formalism based on the study by Casey, Jong, and Gouzé (2006) is recalled in this section: if $x_{i}$ is the protein concentration of the gene $i$ and $\theta_{i}^{j}$ the concentration threshold that characterises the effect of $x_{i}$ on $x_{j}$, the increasing step function $s^{+}: \mathbb{R}_{+} \times \mathbb{R}_{+} \rightarrow\{0,1\}$ is defined:

$$
s^{+}\left(x_{i}, \theta_{j}\right)= \begin{cases}1, & \text { if } x_{i}>\theta_{i}^{j} \\ 0, & \text { if } x_{i}<\theta_{i}^{j}\end{cases}
$$

Accordingly, the step function to express inhibition (or repression) will be: $s^{-}: \mathbb{R}_{+} \times \mathbb{R}_{+} \rightarrow\{0,1\}, s^{-}\left(x_{i}, \theta_{i}^{j}\right)=1-$ $s^{+}\left(x_{i}, \theta_{i}^{j}\right)$. The superscript $j$ indicates the interaction of gene $i$ on gene $j$ of the network.

The production rate of variables $x_{i} \in[0,+\infty), i=1, \ldots, n$ is given by the function $f_{i}: \mathbb{R}_{+}^{n} \rightarrow \mathbb{R}_{+}$:

$$
f_{i}(x)=\sum_{1 \leq l \leq m} \kappa_{i l} D_{i l}(x)
$$

where $\kappa_{i l}>0$ are the synthesis rate parameters and $D_{i l}: \mathbb{R}_{+}^{n} \rightarrow$ $\{0,1\}$ are Boolean-valued functions that express the regulation of variable $x_{i}$ by other variables. The functions $D_{i l}(x)$ may be products of increasing or decreasing step functions $s^{+/-}\left(x_{i}, \theta_{i}^{j}\right)$.

A general form of the system will be:

$$
\dot{x}_{i}=\kappa_{i 0}+f_{i}(x)-\gamma_{i} x_{i}
$$

where $\kappa_{i 0}$ is a basal term, $x=\left(x_{1}, \ldots, x_{n}\right)^{t}>0$ contains the protein concentrations and $\gamma_{i} x_{i}>0$ expresses the degradation rate of protein $x_{i}$.

The thresholds $\theta_{i}^{j}$ define hyperplanes and divide the phase space into regular domains. The regions such that $x_{i}=\theta_{i}^{j}$ for some $i$ and $j$ are called switching domains and form the boundaries of the regular domains. In each regular domain $B$, the vector field is uniquely defined, the synthesis rate is constant $\left(\kappa_{i 0}+\kappa_{i B}\right)$ :

$$
\dot{x}_{i}=\kappa_{i 0}+\kappa_{i B} X-\gamma_{i} x_{i}
$$

and the computation of the system solution, while $x \in B$, is straightforward, with trajectories moving towards the corresponding focal point $\phi_{i B}=\left(\kappa_{i 0}+\kappa_{i B}\right) / \gamma_{i}$. As the trajectory 
reaches the boundary of $B$, the vector field changes and the solution must be computed in the new domain.

In PWA systems, a general property is that a focal point belonging to its own domain (i.e., $\phi_{i B} \in B$ ) is a locally stable steady state. The connection between continuous and piecewise affine models has been closely studied by Casey, Jong, and Gouzé (2006). In the general approach the continuous activation and inhibition functions given by Hill functions are approximated by step functions and lead to a partition of the state space into rectangular domains. In contrast, for the twogene oscillator (1), this approximation leads to a partition defined by a quadratic curve.

\section{B. The PWA system for the two-gene oscillator}

In system (1), the function expressing synthesis of the activator $h_{R}: \mathbb{R}_{+} \rightarrow \mathbb{R}_{+}$is simply:

$$
h_{R}\left(A ; \theta_{1}\right)=\frac{A^{2}}{A^{2}+\theta_{1}},
$$

while the function expressing synthesis of the repressor is given by a composition of two Hill functions $h_{A}: \mathbb{R}_{+}^{2} \rightarrow \mathbb{R}_{+}$:

$$
h_{A}\left((A, R) ;\left(\theta_{0}, \theta_{2}\right)\right)=\frac{A^{2}}{A^{2}+\theta_{0}\left(1+R / \theta_{2}\right)} .
$$

Synthesis of the activator (5) can be straightforwardly approximated by $s_{R}^{+}: \mathbb{R}_{+} \rightarrow\{0,1\}$ :

$$
s_{R}^{+}\left(A ; \theta_{1}\right)= \begin{cases}1, & \text { if } A^{2}>\theta_{1} \\ 0, & \text { if } A^{2}<\theta_{1}\end{cases}
$$

The case of (6) is more complex to deal with. A multiplicative approximation of the form $s^{+}\left(A ; \theta_{1}\right) s^{-}\left(R ; \theta_{2}\right)$ does not faithfully represent the dynamics of the continuous system. So, we chose to approximate the activation part by an increasing step function with a switching value that depends on $R$, as follows $s_{A}^{+}: \mathbb{R}_{+}^{2} \rightarrow\{0,1\}$ :

$$
s_{A}^{+}\left((A, R) ;\left(\theta_{0}, \theta_{2}\right)\right)= \begin{cases}1, & \text { if } A^{2}>\theta_{0}\left(1+R / \theta_{2}\right) \\ 0, & \text { if } A^{2}<\theta_{0}\left(1+R / \theta_{2}\right) .\end{cases}
$$

This is a step function with a coordinate-dependent switching value. This induces a partition of the state space with nonrectangular regular domains, as analyzed in the next section. Applying these approximations, the PWA associated with (1) becomes:

$$
\begin{aligned}
& \frac{d A}{d t}=V_{A} s_{A}^{+}\left((A, R) ;\left(\theta_{0}, \theta_{2}\right)\right)-\gamma_{A} A+r_{\text {bas }} \\
& \frac{d R}{d t}=V_{R} s_{R}^{+}\left(A ; \theta_{1}\right)-\gamma_{R} R .
\end{aligned}
$$

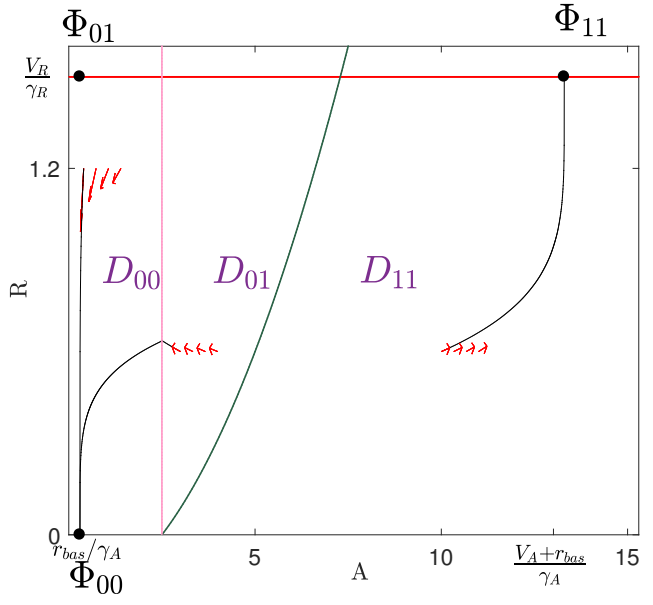

Figure 2. An initial PWA system with activity thresholds $\theta_{0}$ and $\theta_{1}$ equal.

\section{Regular domains and the role of activity thresholds $\theta_{0}$ and $\theta_{1}$ in generating oscillations}

The phase space of the PWA system (9) will be divided in domains by the two threshold hyperplanes $A^{2}=\theta_{1}$ and $A^{2}=$ $\theta_{0}\left(1+R / \theta_{2}\right), R \in\left[0, V_{R} / \gamma_{R}\right]$. However, these domains are no longer rectangles, but instead are defined by a quadratic curve:

$$
\mu(A)=\theta_{2}\left(A^{2}-\theta_{0}\right) / \theta_{0}
$$

According to the original parameter set in Smolen, Baxter, and Byrne (1998) it holds that $\sqrt{\theta_{1}}=\sqrt{\theta_{0}}$, and with this condition the phase space is partitioned into three domains:

$$
\begin{aligned}
& \text { - } \tilde{D}_{00}=\left\{(A, R) \in \mathbb{R}_{+}^{2}: A \in\left[0, \sqrt{\theta_{1}}\right], R \in[0,+\infty)\right\} \\
& \text { - } \tilde{D}_{10}=\left\{(A, R) \quad \in \quad \mathbb{R}_{+}^{2} \quad: \quad A \quad \in\right. \\
& {\left.\left[\sqrt{\theta_{1}}, \sqrt{\theta_{0}\left(1+\left(V_{R} / \gamma_{R}\right) / \theta_{2}\right)}\right], R>\mu(A)\right\} } \\
& \text { - } \tilde{D}_{11}=\left\{(A, R) \in \mathbb{R}_{+}^{2}: A \in\left[\sqrt{\theta_{1}},+\infty\right), R \in[0, \mu(A))\right\}
\end{aligned}
$$

It is not difficult to compute the focal points in each of these three domains and check that, for a large range of parameters, $\phi_{00}, \phi_{01} \in \tilde{D}_{00}$ and $\phi_{11} \in \tilde{D}_{11}$ (see also Fig. 2). Therefore, trajectories in the domain $\tilde{D}_{00}$ converge to the stable solution $\phi_{00}=\left(\phi_{00}^{A}, \phi_{00}^{R}\right)=\left(r_{\text {bas }} / \gamma_{A}, 0\right)$. Similarly, trajectories in $\tilde{D}_{01}$ are attracted by the focal point $\phi_{01}=\left(\phi_{01}^{A}, \phi_{01}^{R}\right)=$ $\left(r_{\text {bas }} / \gamma_{A}, V_{R} / \gamma_{R}\right)$ and trajectories in $\tilde{D}_{11}$ converge to the stable solution $\phi_{11}=\left(\phi_{11}^{A}, \phi_{11}^{R}\right)=\left(\left(V_{A}+r_{\text {bas }}\right) / \gamma_{A}, V_{R} / \gamma_{R}\right)$, see Fig. 2. It is not difficult to see that this state space conformation prevents the existence of periodic oscillations.

A closer look shows that requiring $\sqrt{\theta_{1}}>\sqrt{\theta_{0}}$ will shift the line $A=\sqrt{\theta_{1}}$ to the right of the plane and introduce a fourth region in the state space. Four domains $D_{i j}, i, j \in\{0,1\}$ will be formed under the condition $\sqrt{\theta_{1}}>\sqrt{\theta_{0}}$ :

$$
\begin{aligned}
& \text { - } D_{10}=\left\{(A, R) \in \mathbb{R}_{+}^{2}: A \in\left[0, \sqrt{\theta_{1}}\right], R \in[0, \mu(A))\right\} \\
& \text { - } D_{11}=\left\{(A, R) \in \mathbb{R}_{+}^{2}: A \in\left[\sqrt{\theta_{1}},+\infty\right), R \in[0, \mu(A))\right\}
\end{aligned}
$$


Table I. Focal points, and their location under conditions (a)-(c).

$$
\begin{aligned}
& \begin{array}{c|c}
\hline \phi_{10}=\left(\phi_{10}^{A}, \phi_{10}^{R}\right)=\left(\left(V_{A}+r_{\text {bas }}\right) / \gamma_{A}, 0\right) & D_{11} \\
\hline \phi_{11}=\left(\phi_{11}^{A}, \phi_{11}^{R}\right)=\left(\left(V_{A}+r_{\text {bas }}\right) / \gamma_{A}, V_{R} / \gamma_{R}\right) & D_{01} \\
\hline \phi_{01}=\left(\phi_{01}^{A}, \phi_{01}^{R}\right)=\left(r_{\text {bas }} / \gamma_{A}, V_{R} / \gamma_{R}\right) & D_{00} \\
\hline \phi_{00}=\left(\phi_{00}^{A}, \phi_{00}^{R}\right)=\left(r_{\text {bas }} / \gamma_{A}, 0\right) & D_{10} \\
\hline
\end{array} \\
& \text { - } D_{01}=\left\{(A, R) \in \mathbb{R}_{+}^{2}: A \in\left[\sqrt{\theta_{1}},+\infty\right), R>\mu(A)\right\} \\
& \text { - } D_{00}=\left\{(A, R) \in \mathbb{R}_{+}^{2}: A \in\left[0, \sqrt{\theta_{1}}\right], R>\mu(A)\right\}
\end{aligned}
$$

The focal points in each of the four domains are given in Table I where, for an appropriate but still very large range of parameters, it is possible to place each focal point outside its domain. Indeed, if the focal point $\phi_{00}$ in domain $D_{00}$ is shifted to the domain $D_{10}$ and similarly $\phi_{i j}$ to the following domain in anti-clockwise sense, then the trajectories will be attracted to the corresponding focal point in the neighbor domain. In this way, trajectories are expected to cross the threshold hyperplanes and, since the vector field is uniquely defined in each domain, an oscillatory behavior will eventually be formed by the four trajectory parts.

\section{ANALYSIS OF THE TWO-GENE PWA SYSTEM}

We now choose suitable parameter conditions so that the focal points will be shifted as: $\phi_{00}$ is in the domain $D_{10}, \phi_{10}$ $\in D_{01}, \phi_{11} \in D_{01}$ and $\phi_{01} \in D_{00}$, see Fig. 3 and Table I. For this conformation of the focal points, we will next show that system (9) does admit sustained periodic oscillations.

To prove the existence of a limit cycle we will compute the Poincaré map of system (9) and show that it has a unique fixed point. In each of the four domains $D_{i j}$ there is a map $G_{i j}$ that establishes a correspondence between an entry point at one boundary of $D_{i j}$ and a corresponding exit point after the solution crosses $D_{i j}$. The Poincaré map is given by the composition of these four maps.

\section{A. Parameter conditions}

The configuration listed in Table I is obtained from the conditions on the parameters (a) to (f), stated below and assumed to hold throughout the remainder of this paper. For simplicity, let $\gamma$ denote $\gamma_{R} / \gamma_{A}$ and let $\delta>0$ be small. Condition (a) guarantees the existence of four regions (compare Figs. 2 and 3), while (b) and (c) guarantee, respectively, that $\phi_{11} \in D_{01}$ and $\phi_{00} \in D_{10}$ :
(a) $\sqrt{\theta_{0}}<\sqrt{\theta_{1}}$ and $\gamma_{R}<\gamma_{A}$,
(b) $\sqrt{\theta_{0}}+\delta<\frac{r_{b a s}}{\gamma_{A}}<\sqrt{\theta_{1}}-\delta$
(c) $\sqrt{\theta_{1}}+\delta<\frac{V_{A}+r_{\text {bas }}}{\gamma_{A}}<\sqrt{\theta_{0}\left(1+\frac{V_{R} / \gamma_{R}}{\theta_{2}}\right)}-\delta$

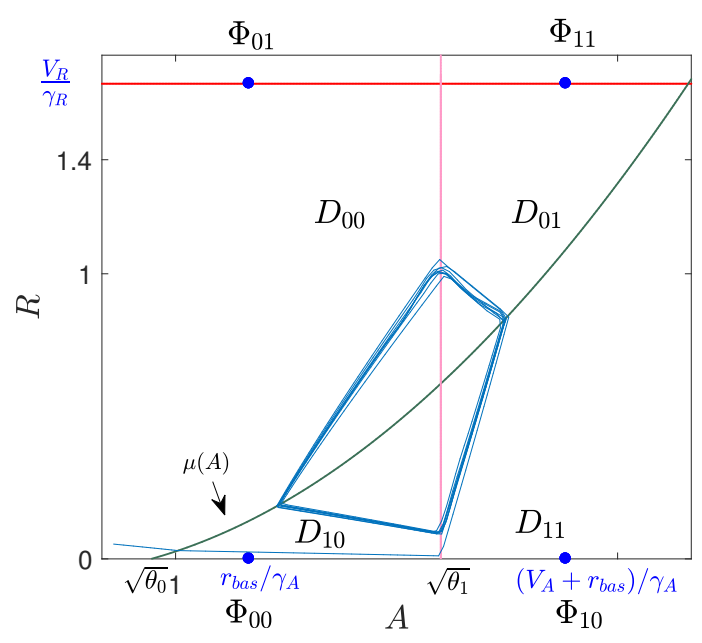

Figure 3. A trajectory of the PWA system following a periodic orbit with parameter values: $V_{A}=0.43 \mathrm{~min}^{-1}, V_{R}=0.3 \mathrm{~min}^{-1}, \gamma_{A}=0.2$ $\mathrm{min}^{-1}, \gamma_{R}=0.18 \mathrm{~min}^{-1}, r_{\text {bas }}=0.3 \mathrm{~min}^{-1}, \theta_{0}=0.7, \theta_{1}=7.84$, $\theta_{2}=0.06$

The inequalities (d)-(f) will be applied below in Lemma 3 to determine the signs of the first and second derivatives of the crossing maps $G_{i j}$, which will help to establish the existence of periodic orbits. Condition (d) guarantees that the map $G_{11}$ has a negative first derivative:

(d) $\left|-\gamma \frac{\mu\left(\sqrt{\theta_{1}}\right)-\phi_{11}^{R}}{\sqrt{\theta_{1}}-\phi_{11}^{A}}\right|>2 \phi_{11}^{A} \frac{\theta_{2}}{\theta_{0}}$.

Conditions (e) establish the size of the first derivatives of the maps $G_{i j}$ at the point $x=\sqrt{\theta_{1}}$ :

(e) i. $\left|-\gamma \frac{\mu\left(\sqrt{\theta_{1}}\right)-\phi_{i j}^{R}}{\sqrt{\theta_{1}}-\phi_{i j}^{A}}+2 \sqrt{\theta_{1}} \frac{\theta_{2}}{\theta_{0}}\right|>1, i j \in\{10,01\}$
ii. $\left|-\gamma \frac{\mu\left(\sqrt{\theta_{1}}\right)-\phi_{i j}^{R}}{\sqrt{\theta_{1}}-\phi_{i j}^{A}}+2 \sqrt{\theta_{1}} \frac{\theta_{2}}{\theta_{0}}\right|<1, i j \in\{00,11\}$.

Conditions (f) establish the sign of the second derivatives of $G_{i j}$ at the point $x=\sqrt{\theta_{1}}$ :

(f) i. $\left|\left(\gamma^{2}+\gamma\right) \frac{\mu\left(\sqrt{\theta_{1}}\right)}{\phi_{11}^{A}-\sqrt{\theta_{1}}}\right|>2 \frac{\theta_{2}}{\theta_{0}}\left(2 \gamma \sqrt{\theta_{1}}+\phi_{11}^{A}-\sqrt{\theta_{1}}\right)$
ii. $\left|\left(\gamma^{2}+\gamma\right) \frac{\mu\left(\sqrt{\theta_{1}}\right)-\phi_{01}^{R}}{\left(\sqrt{\theta_{1}}-\phi_{01}^{A}\right)^{2}}-4 \gamma \frac{\theta_{2}}{\theta_{0}} \frac{\sqrt{\theta_{1}}}{\sqrt{\theta_{1}}-\phi_{01}^{A}}\right|>2 \frac{\theta_{2}}{\theta_{0}}$
iii. $\left(\gamma^{2}+\gamma\right) \frac{\mu\left(\sqrt{\theta_{1}}\right)}{\sqrt{\theta_{1}}-\phi_{00}^{A}}+2 \frac{\theta_{2}}{\theta_{0}}\left(\sqrt{\theta_{1}}-\phi_{00}^{A}\right)>4 \gamma \sqrt{\theta_{1}} \frac{\theta_{2}}{\theta_{0}}$.

It can be easily checked that a set of parameters such as indicated in the caption of Fig. 3 verifies these conditions. 


\section{B. The first return map of the system}

Lemma 1. The following functions characterize the correspondence between entry and exit points of $D_{i j}$ :

$$
G_{i j}(x)=\left(\frac{\sqrt{\theta_{1}}-\phi_{i j}^{A}}{x-\phi_{i j}^{A}}\right)^{\frac{\gamma_{R}}{\gamma_{A}}}\left(\mu(x)-\phi_{i j}^{R}\right)+\phi_{i j}^{R}
$$

for $i, j \in\{0,1\}$ referring to the corresponding domain $D_{i j}$, and defined in the following sets:

- $\left\{\left(x, G_{10}(x)\right) \in \mathbb{R}_{+}^{2}: x \in\left(\phi_{00}^{A}, \sqrt{\theta_{1}}\right), G_{10}(x)<\mu(x)\right\}$

- $\left\{\left(x, G_{11}(x)\right) \in \mathbb{R}_{+}^{2}: x \in\left(\sqrt{\theta_{1}}, \phi_{10}^{A}\right), G_{11}(x)<\mu(x)\right\}$

- $\left\{\left(x, G_{01}(x)\right) \in \mathbb{R}_{+}^{2}: x \in\left(\sqrt{\theta_{1}}, \phi_{11}^{A}\right), G_{01}(x)>\mu(x)\right\}$

- $\left\{\left(x, G_{00}(x)\right) \in \mathbb{R}_{+}^{2}: x \in\left(\phi_{01}^{A}, \sqrt{\theta_{1}}\right), G_{00}(x)>\mu(x)\right\}$

Proof. To obtain these functions, note that in each domain $D_{i j}$, equations (9) can be written as

$$
\frac{d A}{d t}=\gamma_{A}\left(\phi_{i j}^{A}-A\right), \quad \frac{d R}{d t}=\gamma_{R}\left(\phi_{i j}^{R}-R\right) .
$$

Then, the trajectory crossing $D_{i j}$ from a point $\left(A_{0}, R_{0}\right)$ to a point $(A, R)$ is given by:

$$
\left\{\begin{array}{l}
A(t)=\left(A\left(t_{0}\right)-\phi_{i j}^{A}\right) e^{-\gamma_{A}\left(t-t_{0}\right)}+\phi_{i j}^{A} \\
R(t)=\left(R\left(t_{0}\right)-\phi_{i j}^{R}\right) e^{-\gamma_{R}\left(t-t_{0}\right)}+\phi_{i j}^{R}
\end{array}\right.
$$

and solving with respect to time yields the equalities

$$
e^{-\left(t-t_{0}\right)}=\left(\frac{A-\phi_{i j}^{A}}{A_{0}-\phi_{i j}^{A}}\right)^{\frac{1}{\gamma_{A}}}=\left(\frac{R-\phi_{i j}^{R}}{R_{0}-\phi_{i j}^{R}}\right)^{\frac{1}{\gamma_{R}}} .
$$

In addition, for $i j \in\{11,00\}$, we have $A_{0}=\sqrt{\theta_{1}}$ and $R_{0}=$ $\mu\left(A_{0}\right)$. It follows that $R_{0}=G_{i j}\left(A_{0}\right)$. Conversely, for $i j \in$ $\{01,10\}$, let $R=\mu(A)$ and $A=\sqrt{\theta_{1}}$. Then it follows that $R=G_{i j}\left(A_{0}\right)$.

For a trajectory starting at the point $\tilde{P}_{2}=\left(\tilde{A}_{2}, \tilde{R}_{2}\right)=$ $\left(\tilde{A_{2}}, \mu\left(\tilde{A_{2}}\right)\right)$ and returning to another point $P_{2}$, both at the boundary between domains $D_{11}$ and $D_{01}$, the first return map of the system can be computed as follows.

Lemma 2. The Poincaré map of the system (9) is given by the function:

$$
G(x)=G_{11}^{-1} \circ G_{10} \circ G_{00}^{-1} \circ G_{01}(x)
$$

defined on the interval $G:\left[\sqrt{\theta_{1}}, \phi_{10}^{A}\right) \rightarrow\left[\sqrt{\theta_{1}}, \phi_{10}^{A}\right)$.

Proof. Applying the system's solution for each transition in the phase space from $\tilde{P}_{2}$ to $P_{2}$ as it is described in the Proof of Lemma 1 and after some computation follows the Poincaré map of the system.
The first and second derivatives of the Poincaré map $G$, to be used in the next section, can be computed from those of the four crossing maps. The first derivative of each map $G_{i j}$ is given by:

$$
\frac{d G_{i j}}{d x}=\left(\frac{\sqrt{\theta_{1}}-\phi_{i j}^{A}}{x-\phi_{i j}^{A}}\right)^{\gamma}\left[-\gamma \frac{\mu(x)-\phi_{i j}^{R}}{x-\phi_{i j}^{A}}+2 x \frac{\theta_{2}}{\theta_{0}}\right]
$$

and the second derivative is given by:

$$
\frac{d^{2} G_{i j}}{d x^{2}}=\left(\frac{\sqrt{\theta_{1}}-\phi_{i j}^{A}}{x-\phi_{i j}^{A}}\right)^{\gamma}\left[\left(\gamma+\gamma^{2}\right) \frac{\mu(x)-\phi_{i j}^{R}}{\left(x-\phi_{i j}^{A}\right)^{2}}-4 x \gamma \frac{\theta_{2}}{\theta_{0}}+2 \frac{\theta_{2}}{\theta_{0}}\right] .
$$

\section{Existence and uniqueness of limit cycle}

By definition, the fixed points $G\left(x^{*}\right)=x^{*}$ of the Poincaré map indicate the number of periodic orbits of the PWA system (9), and their stability indicates the stability of the orbit. Our main result is thus:

Theorem 1. Assume conditions (a)-(f) hold. Then the first return map (14) of system (9) has a unique fixed point in the open interval $\left(\sqrt{\theta_{1}}, \phi_{0}^{A}\right)$.

To analyse the fixed points of $G$, we will first compute the signs of the first and second derivatives of its components, and show that $G$ is a strictly increasing and concave function. The following properties are easy to check:

Lemma 3. Assume conditions (a)-(f) hold. For each function $G_{i j}, i, j \in\{0,1\}$, the following inequalities hold:

(i) $\frac{d G_{i j}}{d x}>0, \quad i j \in\{10,01\}$

(ii) $\frac{d G_{i j}}{d x}<0, \quad i j \in\{00,11\}$

(iii) $\frac{d^{2} G_{i j}}{d^{2} x}>0, \quad i j \in\{10,00\}$

(iv) $\frac{d^{2} G_{i j}}{d^{2} x}<0, \quad i j \in\{11,01\}$

(v) $\left|\frac{d G_{i j}}{d x}\left(\sqrt{\theta_{1}}\right)\right|>1, \quad i j \in\{10,01\}$

(vi) $\left|\frac{d G_{i j}}{d x}\left(\sqrt{\theta_{1}}\right)\right|<1, \quad i j \in\{00,11\}$.

We can now state the monotonicity properties of $G$ (see also Fig. 4):

Lemma 4. Assume conditions (a)-(f) hold. Then map $G$ defined as in (14) is an increasing and concave function. 


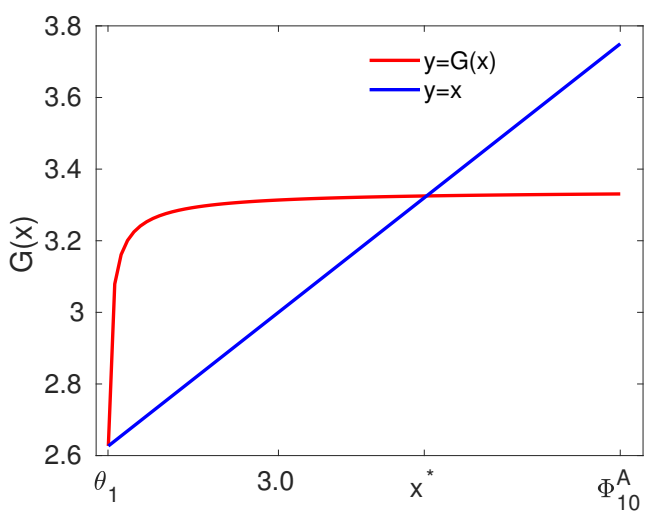

Figure 4. The map $G$, defined in the interval $\left[\sqrt{\theta_{1}}, \phi_{10}^{A}\right)$, computed for the same parameters as in Fig. 3. Its fixed points are given by the intersection with the straight line $y=x$. The point $\sqrt{\theta_{1}}$ corresponds to an unstable fixed point of system (9) while $x^{*}$ corresponds to a locally stable periodic orbit.

Proof. First, from Lemma 3 (i) we have that $G_{11}, G_{00}$ are strictly decreasing functions in their domains, and since they are invertible, $G_{11}^{-1}$ and $G_{00}^{-1}$ are also strictly decreasing. Conversely, by Lemma 3 (ii), the functions $G_{10}, G_{01}$ and their inverse functions are strictly increasing in their domains. Therefore, both $G_{11}^{-1} \circ G_{10}$ and $G_{00}^{-1} \circ G_{01}$ are strictly decreasing, meaning that $G$ is itself strictly increasing as a composition of two decreasing functions.

For the concavity, recall that the inverse $f^{-1}$ of a strictly monotone convex (resp., concave) function $f$ in an open domain $D$ is concave (resp., convex) in its corresponding domain.

Since $G_{10}, G_{00}$ are convex functions and $G_{11}, G_{01}$ are concave (by Lemma 3 (iii) and (iv)), set $f_{1}=G_{00}^{-1} \circ G_{01}$ and note that

$$
f_{1}^{\prime \prime}=\left(G_{00}^{-1}\right)^{\prime \prime} \circ G_{01}\left(G_{01}^{\prime}\right)^{2}+\left(G_{00}^{-1}\right)^{\prime} \circ G_{01} G_{01}^{\prime \prime}>0
$$

and so $f_{1}$ is convex and decreasing as a composition of an increasing and a decreasing function. Similarly

$$
f_{2}^{\prime \prime}=\left[G_{10} \circ f_{1}\right]^{\prime \prime}>0
$$

so it follows that $f_{2}$ is a convex and decreasing function. Lastly, we have that

$$
G^{\prime \prime}=\left[G_{11}^{-1} \circ f_{2}\right]^{\prime \prime}<0
$$

implying that $G$ is concave.

\section{Extremal values $G\left(\sqrt{\theta_{1}}\right)$ and $G\left(\phi_{10}^{A}\right)$}

Before proving our main result, we will evaluate map $G$ at the two endpoints of its interval of definition, that is $\sqrt{\theta_{1}}$ and $\phi_{10}^{A}$. Let us first study the map at $x=\sqrt{\theta_{1}}$, which is equivalent to the point $A=\sqrt{\theta_{1}}, R=\mu\left(\sqrt{\theta_{1}}\right)$ at the boundary of the four regions. At this point, the system (9) must be defined as a differential inclusion and should be written as a convex combination of the vector fields $f_{i j}$ in each of the four domains $D_{i j}$ :

$$
\left[\begin{array}{l}
\frac{d A}{d t} \\
\frac{d R}{d t}
\end{array}\right]=\sum_{i, j=0}^{1} \alpha_{i j} f_{i j}\left(\sqrt{\theta_{1}}, \mu\left(\sqrt{\theta_{1}}\right)\right)
$$

where $\sum_{i, j=0}^{1} \alpha_{i j}=1$. It is easy to see that the zero vector is included in this convex combination, hence the point $\left(\sqrt{\theta_{1}}, \mu\left(\sqrt{\theta_{1}}\right)\right)$ is a Filippov-type equilibrium point of (9) (Filippov, 1960). Moreover, by definition of the partial crossing maps:

$$
\frac{G_{i j}(x)-\phi_{i j}^{R}}{\mu(x)-\phi_{i j}^{R}}=\left(\frac{\sqrt{\theta_{1}}-\phi_{i j}^{A}}{x-\phi_{i j}^{A}}\right)^{\frac{\gamma_{R}}{\gamma_{A}}}
$$

it follows that $G_{i j}\left(\sqrt{\theta_{1}}\right)=\mu\left(\sqrt{\theta_{1}}\right)$ for all $i j$, hence $G\left(\sqrt{\theta_{1}}\right)=$ $\sqrt{\theta_{1}}$ and $\sqrt{\theta_{1}}$ is a fixed point of the first return map. However, the combination of Lemma 3 (v) and (vi) implpies that $G^{\prime}\left(\sqrt{\theta_{1}}\right)>0$, which in turn implies that $\sqrt{\theta_{1}}$ corresponds to an unstable fixed point of (9).

At the other endpoint $x=\phi_{10}^{A}$, by definition of focal point, it follows that $G\left(\phi_{10}^{A}\right)<\phi_{10}^{A}$, since $\phi_{11}^{A}=\phi_{10}^{A}$ and for $A_{0} \in D_{11}$ $d A / d t=-\gamma_{A}\left(\phi_{11}^{A}-A\right)$ implies $A\left(t ; A_{0}\right)<\phi_{10}^{A}$ for all $t$ and $A<$ $A_{0}$.

\section{Proof of Theorem 1}

We want to show that there exists a unique $\sqrt{\theta_{1}}<x^{*}<\phi_{10}^{A}$ such that $x^{*}=G\left(x^{*}\right)$. From the above discussion, $G$ is strictly increasing and concave in $\left[\sqrt{\theta_{1}}, \phi_{0}^{A}\right)$ and satisfies $G\left(\sqrt{\theta_{1}}\right)=$ $\sqrt{\theta_{1}}$ and $G\left(\phi_{10}^{A}\right)<\phi_{10}^{A}$. Therefore $G$ intersects the line $y=x$ exactly once in the open interval.

To show that this fixed point $x^{*}$ is stable, i.e. $d G / d x\left(x^{*}\right)<$ 1 , consider the function $L(x)=G(x)-x$ on $\left[\sqrt{\theta_{1}}, \phi_{10}^{A}\right)$. This function has exactly two zeros on this interval, $\sqrt{\theta_{1}}$ and $x^{*}$. By the Intermediate Value Theorem, $L^{\prime}(x)$ has one zero at $r \in\left(\sqrt{\theta_{1}}, x^{*}\right)$ and $L$ is decreasing between $r$ and $x^{*}+\delta$ for some $\delta>0$ (since $G(x)$ is continous and $G(x)<x$ in $\left(x^{*}, \phi_{10}^{A}\right)$ ). Therefore, $L^{\prime}(x)=G^{\prime}(x)-1<0$ and $0<G^{\prime}(x)<1$ for all $x \in\left(r, x^{*}+\delta\right)$.

\section{Focal points shift due to basal synthesis rate for the repressor}

This Section generalizes system (9) by adding a basal production rate to the repressor equation. This result will be useful later on in Section IV C, where we need to characterize the shift in the focal points due to the network coupling. Define the following PWA system associated with (9): 


$$
\begin{aligned}
& \frac{d A}{d t}=V_{A} s_{A}^{+}\left((A, R) ;\left(\theta_{0}, \theta_{2}\right)\right)-\gamma_{A} A+r_{\text {bas }} \\
& \frac{d R}{d t}=V_{R} s_{R}^{+}\left(A ; \theta_{1}\right)-\left(\gamma_{R}+\beta\right) R+\beta C,
\end{aligned}
$$

with $s_{A}^{+}, s_{R}^{+}$, as defined in Section II B; $\beta>0$ is a constant that controls the shift of $\phi_{i j}^{R}$ coordinates in the phase space and $C>0$ expresses a basal term that remains constant. The other parameters are those of system (9).

The system domains are defined as in Section IIC and the coordinates $\phi_{i j}^{R}$ can be computed as a shift of the corresponding focal points $R$-coordinates of system (9):

$$
\begin{gathered}
\phi_{00}^{R}=\phi_{10}^{R}=\frac{\beta C}{\gamma_{R}+\beta} \\
\phi_{01}^{R}=\phi_{11}^{R}=\frac{V_{R}+\beta C}{\gamma_{R}+\beta}
\end{gathered}
$$

Note that the $\phi_{i j}^{A}$ coordinates remain the same as in Table I.

Additionally to parameter conditions (a)-(f), we next introduce conditions on $\beta$, for which the location of the focal points (the domain in which $\phi_{i j}$ is located) is the same as in Table I); these conditions will imply that the system (18) also admits a unique periodic solution as shown in Section III C. Introduce first the notation:

$$
a_{\text {bas }}=\frac{r_{b a s}}{\gamma_{A}}, \quad a_{\max }=\frac{V_{A}+r_{b a s}}{\gamma_{A}} .
$$

For system (18), the following holds:

(i) $\phi_{00} \in D_{10}$ if and only if $\phi_{00}^{R}<\mu\left(a_{b a s}\right)$,

(ii) $\phi_{11} \in D_{01}$ if and only if $\phi_{11}^{R}>\mu\left(a_{\max }\right)$.

From (19), these inequalities become:

(i) $\beta<\beta_{1}(C):=\mu\left(a_{\text {bas }}\right) \gamma_{R} /\left(C-\mu\left(a_{\text {bas }}\right)\right)$, whenever $C>$ $\mu\left(a_{\text {bas }}\right)$,

(ii) $\beta<\beta_{2}(C):=\left(\mu\left(a_{\max }\right) \gamma_{R}-V_{R}\right) /\left(C-\mu\left(a_{\max }\right)\right)$, whenever $C<\mu\left(a_{\max }\right)$.

Combining these two inequalities, yields the following condition on $\beta$ :

$$
\beta<\left\{\begin{array}{lr}
\beta_{1}(C), & \text { if } C>\mu\left(a_{\max }\right) \\
\beta_{2}(C), & \text { if } C<\mu\left(a_{\text {bas }}\right) \\
\min \left\{\beta_{1}(C), \beta_{2}(C)\right\}, & \text { if } \mu\left(a_{\text {bas }}\right)<C<\mu\left(a_{\max }\right)
\end{array}\right.
$$

If $\beta$ does not satisfy these conditions, it follows that the shift in $R$-coordinates of the focal points induces a change in the dynamic of system (18) from sustained oscillations to stability: for $C>\mu\left(a_{\max }\right)$, if the condition on $\beta$ is not satisfied, then $\phi_{00}$ shifts to its corresponding domain $D_{00}$ and becomes locally stable solution for the system (18). Analogously, for $C<\mu\left(a_{b a s}\right)$ and the corresponding condition on $\beta$ not satisfied, $\phi_{11}$ changes location to $D_{11}$ and becomes steady state.
Lastly, for $\mu\left(a_{b a s}\right)<C<\mu\left(a_{\text {max }}\right), \phi_{00}$ shifts to $D_{00}$ for $\beta>\beta_{1}$ and $\phi_{11}$ to $D_{11}$ for $\beta>\beta_{2}$. In either of these three cases, the periodic solution disappears. This observation will be used in Section IV C to show stability of a network under some conditions.

\section{NETWORK DYNAMICS OF $N$ COUPLED TWO-GENE OSCILLATORS}

Motivated by the synchronization of mammalian circadian clocks in tissues and organs, we will consider a network of $N$ two-gene oscillators interconnected according to three different topologies. Experiments have shown that the mammalian suprachiasmatic nucleus $(\mathrm{SCN})$, that consists of neurons and is located in the brain, is the central synchronizer of the circadian rhythms, (Ralph et al., 1990). In parallel, in cultured cells and tissue explants, there have been observed self-sustained and autonomous circadian clocks (Balsalobre, Damiola, and Schibler, 1998), (Yamazaki et al., 2000). The suprachiasmatic nucleus $(\mathrm{SCN})$ is the central circadian synchroniser, sending signals to the rest of the brain and to the pheripheral clocks in the body. For the form of this interaction between the $\mathrm{SCN}$ and the peripheral clocks, we can consider diffusive exchanges between cells (Silver et al., 1996).

In the context of the hierarchical organisation of the circadian system, we explore a network $N$ two-gene oscillators coupled through diffusion terms. In a first case, we consider one PWA system (9) to represent the SCN and the other $N-1$ identical systems as peripheral clocks, leading to an "one-to-all" or "star" topology where oscillator $i=1$ is connected to all others, as in Fig. 5. In a second case, we consider all oscillators to play identical roles, as in a homogeneous tissue, and study an "all-to-all" topology where each oscillator is connected to all others. As an intermediate case, we will consider randomly chosen connections, within certain parameters.

\section{A. PWA oscillator network and location of focal points}

Consider $N$ identical oscillators of the form (9), with coordinates $\left(A_{i}, R_{i}\right) i \in\{1,2, \ldots, N\}$, coupled through $R_{i}$ according to some topology $L$ and a diffusion parameter $\kappa$ :

$$
\begin{aligned}
& \frac{d A_{i}}{d t}=V_{A} s_{A, R}^{+}\left(A_{i}, R_{i} ; \theta_{0}, \theta_{2}\right)-\gamma_{A} A_{i}+r_{\text {bas }} \\
& \frac{d R_{i}}{d t}=V_{R} s_{A}^{+}\left(A_{i} ; \theta_{1}\right)-\gamma_{R} R_{i}+\kappa \sum_{j \in \mathscr{L}}\left(R_{j}-R_{i}\right), i=1, \ldots, N,
\end{aligned}
$$

where the set $\mathscr{L}$ contains all systems $j$ that are connected to $i$. It will be useful to define the vectors $A=\left(A_{1}, \ldots, A_{N}\right)^{\prime}$ and $R=\left(R_{1}, \ldots, R_{N}\right)^{\prime}$, as well as the state of the coupled system as $X=\left(A_{1}, R_{1}, \ldots, A_{i}, R_{i}, \ldots, A_{N}, R_{N}\right)^{\prime} \in \mathbb{R}_{\geq 0}^{2 N}$. We further define

$$
s h_{A, R}=\left[s_{A}^{+}\left(\left(A_{1}, R_{1}\right) ;\left(\theta_{0}, \theta_{2}\right)\right), \ldots, s_{A}^{+}\left(\left(A_{n}, R_{n}\right) ;\left(\theta_{0}, \theta_{2}\right)\right)\right]^{\prime}
$$


and

$$
s h_{A}=\left[s_{A}^{+}\left(A_{1} ; \theta_{1}\right), \ldots, s_{A}^{+}\left(A_{n} ; \theta_{1}\right)\right]^{\prime}
$$

to be $N$-dimensional Boolean vector functions, evaluated according to the state $X$. The coupled system (22) can be rewritten as:

$$
\begin{aligned}
& \frac{d A}{d t}=V_{A} s h_{A, R}-\Gamma_{A} A+r_{b a s} \\
& \frac{d R}{d t}=V_{R} s h_{A}-\left(\Gamma_{R}+L\right) R
\end{aligned}
$$

where $\Gamma_{A}, \Gamma_{R}$ are diagonal $N \times N$ matrices with elements $\gamma_{A}$, $\gamma_{R}>0$ respectively, and (by abuse of notation) $r_{\text {bas }}$ is an $N$ dimensional vector with elements $r_{\text {bas }} . L$ is the symmetric $N \times$ $N$ Laplacian matrix of the connection topology, to be defined for each case.

Analogously to Section IIC, we define the domains of system (23) as $D_{C}=D_{c_{1}} \times \cdots \times D_{c_{N}}$, where $c_{i}$ belongs to $\{00,01,10,11\}$, so that each $D_{c_{i}}$ corresponds to a regular domain of the 2-dimensional system (9). The focal points for each domain $D_{C}$ are denoted by $\phi_{C}=\left(\tilde{A}^{C}, \tilde{R}^{C}\right)^{\prime}$ and they are given by:

$$
\begin{aligned}
& \tilde{A}^{C}=V_{A} \Gamma_{A}^{-1} s h_{A, R}\left[D_{C}\right]+\Gamma_{A}^{-1} r_{\text {bas }} \\
& \tilde{R}^{C}=V_{R}\left(\Gamma_{R}+L\right)^{-1} s h_{A}\left[D_{C}\right]
\end{aligned}
$$

where $s h_{A, R}\left[D_{C}\right]$ and $s h_{A}\left[D_{C}\right]$ are the synthesis rate vectors evaluated at domain $D_{C}$. As before, the location of the focal points determines the dynamics of the system. In Section III C we have shown that the (single) two-gene oscillator (9) admits a unique periodic solution for certain parameter conditions. This periodic solution, which we call $\left(\varphi_{A}(t), \varphi_{R}(t)\right)$, extends to naturally as a solution to the coupled system with $\varphi_{A}^{i}(t)=\varphi_{A}(t)$ and $\left.\left.\varphi_{R}^{i}(t)\right)=\varphi_{R}(t)\right)$ for all $i$. This can be seen by taking identical initial conditions for all systems $i$. Intriguingly, our analysis shows that diffusive coupling generates new locally stable steady states for the coupled system. Notice that only the coordinates $\tilde{R}^{C}$ depend on the coupling parameter $\kappa$, as well as on the number $N$ of oscillators, while $\tilde{A}^{C}$ are independent of the coupling topology and can be computed for $i=1, \ldots, N$ as follows

$$
\tilde{A}_{i}^{C}= \begin{cases}a_{\text {bas }}, & D_{C_{i}} \in\left\{D_{00}, D_{10}\right\} \\ a_{\text {max }}, & D_{C_{i}} \in\left\{D_{11}, D_{01}\right\}\end{cases}
$$

where we used notation (20). This implies that regular domains $D_{C}$ whose product includes $\left\{D_{01}, D_{10}\right\}$ for some $i$ cannot contain their own focal point $\phi_{C}$. Conversely, for regular domains $D_{C}$ which are products of $D_{00}$ and $D_{11}$ only, the location of the focal points depends on the value of each $\tilde{R}_{i}^{C}$ relative to the curve $\mu(A)$ (see Fig. 3), as stated in the next Lemma.

Lemma 5. For each oscillator $i$, the following hold:

(a) Let $D_{c_{i}}=D_{00}, \tilde{R}_{i}^{C} \in D_{00}$ if and only if $\tilde{R}_{i}^{C}>\mu\left(a_{\text {bas }}\right)$,

(b) Let $D_{c_{i}}=D_{11}, \tilde{R}_{i}^{C} \in D_{11}$ if and only if $\tilde{R}_{i}^{C}<\mu\left(a_{\max }\right)$.

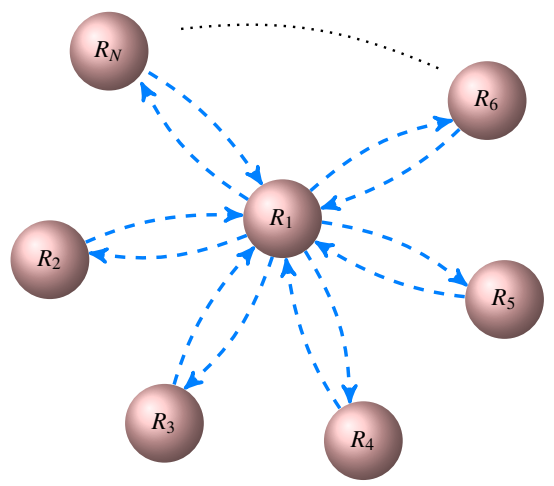

Figure 5. Star topology scheme: coupling $N$ two-gene oscillators of the form (9), through variable $R$.

Consider a regular domain $D_{C}$ with $D_{c_{i}} \in\left\{D_{00}, D_{11}\right\}$ for all $i=1, \ldots, N$. Then $\phi_{C}=\left(\tilde{A}^{C}, \tilde{R}^{C}\right)^{\prime} \in D_{C}$ if and only if either (a) or (b) are satisfied for each $i$.

The inequalities (a) and (b) follow directly from the expressions (25) for $\tilde{A}^{C}$ and the geometry of the phase space. The result on $\phi_{C}$ is an immediate consequence of (a) and (b).

\section{B. Star Topology}

This simplified interconnection scheme naturally assumes that $S C N$ (represented by $R_{1}$ ) is connected to all other peripheral clocks, but the latter do not communicate among themselves, as in the network depicted in Fig. 5. The corresponding Laplacian matrix $L$ is defined as

$$
L=\kappa\left(\begin{array}{ccccc}
N-1 & -1 & -1 & \ldots & -1 \\
-1 & 1 & 0 & \ldots & 0 \\
-1 & 0 & 1 & \ldots & 0 \\
\vdots & & & & \vdots \\
-1 & 0 & 0 & \ldots & 1
\end{array}\right)
$$

In each of the $4^{N}$ regular domains of the coupled system the coordinates of the focal points can be computed as in (24), where the matrix $\left(\Gamma_{R}+L\right)^{-1}$ is strictly positive and has the form

$$
\left[\begin{array}{ccccc}
Q & S & S & \cdots & S \\
S & P & T & \cdots & T \\
S & T & P & \cdots & T \\
\vdots & \vdots & \vdots & & \vdots \\
S & T & T & \cdots & P
\end{array}\right]
$$


where the entries are given by:

$$
\begin{aligned}
S & =\frac{\kappa}{\gamma_{R}\left(\gamma_{R}+N \kappa\right)} \\
Q & =\frac{\gamma_{R}}{\gamma_{R}\left(\gamma_{R}+N \kappa\right)}+S \\
T & =\frac{\kappa}{\gamma_{R}+\kappa} S \\
P & =\frac{\left(\gamma_{R}+\kappa\right)^{2}+(N-2) \gamma_{R} \kappa}{\kappa^{2}} T .
\end{aligned}
$$

Based on Lemma 5, we can now show that new steady states may be generated due to the coupling scheme. In addition, using (28), we can estimate the number of these new steady states in terms of the coupling parameter and the number of oscillators in the network. Introduce the notation:

$$
D_{00} \times\left(D_{11}^{m} \times D_{00}^{N-m-1}\right)_{p r m}, \quad D_{11} \times\left(D_{11}^{m-1} \times D_{00}^{N-m}\right)_{p r m}
$$

to denote products of $m$ domains of the form $D_{11}$ and $N-m$ of the form $D_{00}$, where only the first component $D_{c 1}$ is fixed, and the other components are any permutation of the remaining domains. For example, in the case $N=3, m=2$ we have: $D_{00} \times D_{11} \times D_{11}$ and $D_{11} \times D_{11} \times D_{00}, D_{11} \times D_{00} \times D_{11}$.

Theorem 2. Consider system (23) in a star-coupled topology, with L defined as in (26). Assume that conditions (a)-(f) hold.

Case (i) Define the positive integers:

$$
\begin{aligned}
& m_{\min }=\left\lceil\frac{1}{T V_{R}} \mu\left(a_{\text {bas }}\right)\right\rceil \\
& m_{\max }=\left\lfloor\frac{1}{T V_{R}} \mu\left(a_{\max }\right)-\frac{\left(\gamma_{R}+\kappa\right)^{2}+(N-2) \gamma_{R} \kappa}{\kappa^{2}}+1\right\rfloor .
\end{aligned}
$$

If $m_{\min }<m_{\max }$, then system (23) has exactly $\sum_{m=m_{\min }}^{m_{\max }}(N-$ $1) /(m !(N-m-1) !)$ locally stable steady states corresponding to focal points $\phi_{C} \in D_{C}=D_{00} \times\left(D_{11}^{m} \times D_{00}^{N-m-1}\right)_{\text {prm }}$.

Case (ii) Define the positive integers:

$m_{\text {min }}=\left\lceil\frac{1}{T V_{R}} \mu\left(a_{b a s}\right)-\frac{\gamma_{R}}{\kappa}\right\rceil$

$m_{\max }=\left\lfloor\frac{1}{T V_{R}} \mu\left(a_{\max }\right)-\frac{\gamma_{R}}{\kappa}-\frac{1}{\gamma_{R}+\kappa} \frac{1}{T} \max \left\{\frac{\gamma_{R}}{V_{R}} \mu\left(a_{\max }\right), 1\right\}\right\rfloor$.

If $m_{\min }<m_{\max }$, then system (23) has exactly $\sum_{m=m_{\min }}^{m_{\max }}(N-$ $1) ! /((m-1) !(N-m) !)$ locally stable steady states corresponding to focal points $\phi_{C} \in D_{C}=D_{11} \times\left(D_{11}^{m-1} \times D_{00}^{N-m}\right)_{\text {prm }}$.

Case (iii) Let

$$
\hat{\kappa}=\left\{\begin{array}{l}
\max \left\{\frac{\gamma_{R}\left(\mu\left(a_{\text {max }}\right) \gamma_{R}-V_{R}\right)}{V_{R}-N \gamma_{R} \mu\left(a_{\text {max }}\right)}, \frac{\gamma_{R}^{2} \mu\left(a_{\text {bas }}\right)}{V_{R}-N \gamma_{R} \mu\left(a_{\text {bas }}\right)}\right\}, \text { if } N \leq N^{*} \\
\frac{V_{R}}{\mu\left(a_{\text {max }}\right)-\mu\left(a_{\text {bas }}\right)}-\gamma_{R}, \text { if } N>N^{*}
\end{array}\right.
$$

with $N^{*}=\left\lceil\frac{V_{R}}{\gamma_{R} \mu\left(a_{\text {bas }}\right)}-\frac{\gamma_{R}\left(\mu\left(a_{\max }\right)-\mu\left(a_{\text {bas }}\right)\right)}{V_{R}-\gamma_{R}\left(\mu\left(a_{\max }\right)-\mu\left(a_{\text {bas }}\right)\right)}\right\rceil$. Then no new steady states exist for any $\kappa \leq \hat{\kappa}$.
Proof. First, recall that a focal point $\phi_{C}$ is a locally stable state if and only if it belongs to its own domain $D_{C}$ : since the eigenvalues of the matrices $-\Gamma_{A}$ and $-\left(\Gamma_{R}+L\right)$ have strictly negative real part, the Hurwitz condition holds, hence the focal points that lie in their own domains are locally asymptotically stable. Second, Lemma 5 provides the conditions required to guarantee $\phi_{C} \in D_{C}$. Consider a general domain $D_{C}$ consisting of the product of $m$ regions of the form $D_{11}$ and $N-m$ of the form $D_{00}$, in any order. Since oscillator 1 has a special role, we need to consider two cases, depending on whether $c_{1}=00$ or $c_{1}=11$ :

Case (i) $D_{C}=D_{00} \times\left(D_{11}^{m} \times D_{00}^{N-m-1}\right)_{p r m}$. In this case, the vector $s h_{A}\left[D_{C}\right]$ has $m$ entries equal to 1 , and (27) and (24) lead to

$$
\begin{aligned}
& \tilde{R}_{1}^{C}=m S V_{R} \\
& \tilde{R}_{i}^{C}= \begin{cases}(P+(m-1) T) V_{R}, & \text { if } s h_{A, i}=1, i=2, \ldots, N \\
m T V_{R}, & \text { if } s h_{A, i}=0, i=2, \ldots, N .\end{cases}
\end{aligned}
$$

By Lemma 5, the conditions to guarantee $\phi_{C} \in D_{C}$ become

$$
\begin{aligned}
m S V_{R} & >\mu\left(a_{\text {bas }}\right) \\
(P+(m-1) T) V_{R} & <\mu\left(a_{\text {max }}\right) \\
m T V_{R} & >\mu\left(a_{\text {bas }}\right)
\end{aligned}
$$

From (28), it follows that $S>T$ so, combining these inequalities leads to

$$
\frac{1}{T V_{R}} \mu\left(a_{\text {bas }}\right)<m<1+\frac{1}{T V_{R}} \mu\left(a_{\max }\right)-\frac{P}{T},
$$

and (28) shows that $P / T=\left(\left(\gamma_{R}+\kappa\right)^{2}+(N-2) \gamma_{R} \kappa\right) / \kappa^{2}$ which yields $m_{\min }<m<m_{\max }$. If there exists an integer $m$ in this interval, this means that any choice of $m$ oscillators in $D_{11}$ implies that the corresponding focal point belongs to $D_{C}$. Now, there are several ways of choosing $m$ oscillators among the $N$. In this first case, $D_{c_{1}}=D_{00}$ is fixed, so the formula is " $N-1$ choose $m$ ", that is $(N-1) ! /(m !(N-1-m) !)$ ways for each $m$. Since there is one focal point for each of these choices and this is valid for any integer $m$ in the interval $\left[m_{\min }, m_{\max }\right]$, obtain the given sum for the total number of new steady states.

Case (ii) $D_{C}=D_{11} \times\left(D_{11}^{m-1} \times D_{00}^{N-m}\right)_{p r m}$. The coordinates $\tilde{R}^{C}$ of the focal points are now given by

$$
\begin{aligned}
& \tilde{R}_{1}^{C}=(Q+(m-1) S) V_{R} \\
& \tilde{R}_{i}^{C}= \begin{cases}(S+P+(m-2) T) V_{R}, & \text { if } s h_{A, i}=1, i=2, \ldots, N \\
(S+(m-1) T) V_{R}, & \text { if } s h_{A, i}=0, i=2, \ldots, N .\end{cases}
\end{aligned}
$$

Applying Lemma 5 yields,

$$
\begin{array}{r}
(Q+(m-1) S) V_{R}<\mu\left(a_{\text {max }}\right) \\
(S+P+(m-2) T) V_{R}<\mu\left(a_{\text {max }}\right) \\
(S+(m-1) T) V_{R}>\mu\left(a_{\text {bas }}\right) .
\end{array}
$$

Combining the first two inequalities

$$
m<\min \left\{1+\frac{1}{S V_{R}} \mu\left(a_{\max }\right)-\frac{Q}{S}, 2+\frac{1}{T V_{R}} \mu\left(a_{\max }\right)-\frac{P+S}{T}\right\} \text {, }
$$


using (28) to obtain $Q / S=1+\gamma_{R} / \kappa,(S+P) / T=2+\gamma_{R} / \kappa+$ $1 /(\kappa S)$

$$
m<\min \left\{\frac{1}{S V_{R}} \mu\left(a_{\max }\right)-\frac{\gamma_{R}}{\kappa}, \frac{1}{T V_{R}} \mu\left(a_{\max }\right)-\frac{\gamma_{R}}{\kappa}-\frac{1}{\kappa S}\right\},
$$

and noticing that $T / S=\kappa /\left(\gamma_{R}+\kappa\right)=1-\gamma_{R} /\left(\gamma_{R}+\kappa\right)$ yields $m_{\min }<m<m_{\max }$. In this case, the first oscillator is fixed at $D_{c_{1}}=D_{11}$ so the formula becomes " $N-1$ choose $m-1$ ", that is $(N-1) ! /((m-1) !(N-m) !)$ for each $m$.

We note that if the inequalities

$$
\begin{aligned}
Q V_{R} & <\mu\left(a_{\max }\right) \\
(S+P+T) V_{R} & >\mu\left(a_{\text {max }}\right) \\
S V_{R} & >\mu\left(a_{\text {bas }}\right)
\end{aligned}
$$

are satisfied and $m_{\min } \geq m_{\max }$ for the values defined in (29), then there is exactly one locally stable steady state for the system (23), that is of the form $D_{C}=D_{11} \times\left(D_{00}\right)^{N-1}$. This derives from the inequalities satisfied in Case (ii), for $m=1$.

Case (iii) To prove the last statement of Theorem 2, observe that $\hat{\kappa}$ is the value of $\kappa$ that renders $m_{\min }>m_{\max }$ for the values as defined by (29) and (31), that is the intersection of

$$
\begin{aligned}
\left\{\frac{1}{T V_{R}} \mu\left(a_{\max }\right)-\frac{P}{T}\right. & \left.+1<\frac{1}{T V_{R}} \mu\left(a_{\text {bas }}\right)\right\} \\
& \cap\left\{Q V_{R}>\mu\left(a_{\max }\right)\right\} \\
& \cap\left\{S V_{R}<\mu\left(a_{\text {bas }}\right)\right\}
\end{aligned}
$$

gives an interval for $\kappa$ such that no focal point belongs to its own domain. Solving $\mu\left(a_{\max }\right) / T V_{R}-P / T+1<$ $\mu\left(a_{b a s}\right) / T V_{R}$ with respect to $\kappa$ and using (28) yields the value of $\hat{\kappa}_{c}=V_{R} /\left(\mu\left(a_{\max }\right)-\mu\left(a_{b a s}\right)\right)-\gamma_{R}$, which is constant $\forall N$. Solving $S V_{R}<\mu\left(a_{\text {bas }}\right)$ with respect to $N$ for $\hat{\kappa}_{c}$ to obtain

$$
N^{*}=\left\lceil\frac{V_{R}}{\gamma_{R} \mu\left(a_{\text {bas }}\right)}-\frac{\gamma_{R}\left(\mu\left(a_{\max }\right)-\mu\left(a_{\text {bas }}\right)\right)}{V_{R}-\gamma_{R}\left(\mu\left(a_{\max }\right)-\mu\left(a_{\text {bas }}\right)\right)}\right\rceil,
$$

that is the maximum value of $N$ for which, the value of $\hat{\kappa}$ is given by solving $\left\{Q V_{R}>\mu\left(a_{\max }\right)\right\} \cap\left\{S V_{R}<\mu\left(a_{b a s}\right)\right\}$ with respect to $\kappa$.

Example 1: $N=2$. System (23) has 16 regular domains and the corresponding focal points are given in Table (II).

In this case, for a coupling constant $\kappa=0.25$, and the other parameters as in Fig. 3, we have $S=2.0425, T=1.1875, Q=$ $3.5131, P=3.5131, \mu\left(a_{\text {bas }}\right)=0.1336, \mu\left(a_{\max }\right)=1.0865$, $\hat{\kappa}=0.21$ and the bounds (29) and (30):

$$
\begin{gathered}
0.375<m<1.0914 \\
Q V_{R}=1.05<\mu\left(a_{\max }\right) \text { and } S V_{R}=0.6127>\mu\left(a_{\text {bas }}\right) .
\end{gathered}
$$

According to Theorem $2, m=1$ thus implying at most $1 ! /(1 ! 0 !)=1$ new steady state in $D_{0011}$ and $1 ! /(0 ! 1 !)=1$ new steady state in $D_{1100}$, in agreement with Table II. The value of $\hat{\kappa}$ also agrees with simulations, see Fig. 7.

Example 2: $N=10$. Similarly, the computations of the $\mathrm{m}$ bounds in Theorem 2 case (i) yield $1.4783<m<4.3013$, thus
Table II. Focal points of 2 systems coupled and their corresponding location in the domains for $\kappa=0.25$.

\begin{tabular}{l|l|l|l}
\hline$\Phi_{1010}$ & $D_{1111}$ & $\Phi_{1011}$ & $D_{1111}$ \\
\hline$\Phi_{1001}$ & $D_{1100}$ & $\Phi_{1000}$ & $D_{1110}$ \\
\hline$\Phi_{1110}$ & $D_{1111}$ & $\Phi_{1111}$ & $D_{0101}$ \\
\hline$\Phi_{1101}$ & $D_{0100}$ & $\Phi_{1100}$ & $D_{1100}$ \\
\hline$\Phi_{0110}$ & $D_{0011}$ & $\Phi_{0111}$ & $D_{0001}$ \\
\hline$\Phi_{0101}$ & $D_{0000}$ & $\Phi_{0100}$ & $D_{0000}$ \\
\hline$\Phi_{0010}$ & $D_{1011}$ & $\Phi_{0011}$ & $D_{0011}$ \\
\hline$\Phi_{0001}$ & $D_{0000}$ & $\Phi_{0000}$ & $D_{1010}$ \\
\hline
\end{tabular}

$m_{\min }=2$, and $m_{\max }=4$. We expect $\sum_{j=2}^{4}(9) ! /((j) !(9-j) !)=$ 246 steady states in domains of the form $D_{00} \times\left(D_{11}\right)^{4} \times$ $\left(D_{00}\right)^{N-5}$. Applying the inequalities of case (ii) to obtain $0.7583<m<3.5813$, with $m_{\min }=1$ and $m_{\max }=3$, we expect $\sum_{j=1}^{3}(9) ! /((j-1) !(10-j) !)=46$ steady states in domains of the form $D_{11} \times\left(D_{11}\right)^{3} \times\left(D_{00}\right)^{N-3}$. Our estimations are exact as it is illustrated by comparison with numerical computations in Fig. 6.

\section{Star-coupled network stabilization}

In this section we show that the star topology does not admit "mixed" dynamics: if any of the two-dimensional systems converges to a steady state, then all the network stabilizes.

Theorem 3. Consider system (23) in a star-coupled topology, with $L$ defined as in (26). Assume that conditions $(a)-(f)$ hold. If any $2 D$ system $k$ converges to a steady state (away from the domain boundaries), then the full network converges to a steady state.

Proof. Suppose that 2D system $k \neq 1$, converges a to steady state in a regular domain, $\tilde{R}_{k}=$ constant. Then, from (25), there are two possible values for $\tilde{A}_{k}$ :

$$
\tilde{A}_{k}=\left\{\begin{array}{l}
a_{\text {bas }}, \quad \text { which implies } s h_{A, R}=0 \text { and } s h_{A}=0 \\
a_{\max }, \quad \text { which implies } s h_{A, R}=1 \text { and } s h_{A}=1 .
\end{array}\right.
$$

For simplicity we denote $\delta_{j}=\left(s h_{A}\right)_{j} \in\{0,1\}$.

By assumption, $\tilde{R}_{k}$ must remain constant (or within an interval of length $\varepsilon$ as small as desired), $\delta_{k}$ remains fixed, and $\dot{R}_{k} \approx 0$, thus (22) implies:

$$
\kappa L_{k k} \tilde{R}_{k}+\gamma_{R} \tilde{R}_{k} \approx V_{R} \delta_{k}(t)+\kappa \sum_{j \in \mathscr{L}} \tilde{R}_{j}
$$

Since oscillator $k$ is only connected to system $j=1$, from (24) and (26), it follows that $L_{k k}=1$ and $\sum_{j \in \mathscr{L}} \tilde{R}_{j}=\tilde{R}_{1}$. Solving (33) with respect to $\tilde{R}_{1}$ gives :

$$
\tilde{R}_{1} \approx \frac{\kappa+\gamma_{R}}{\kappa} \tilde{R}_{k}-\frac{V_{R}}{\kappa} \delta_{k}(t)
$$

Since both $\tilde{R}_{k}$ and $\delta_{k}(t)$ are fixed, the latter implies that $\tilde{R}_{1}=$ constant, and so $\left(A_{1}(t), R_{1}(t)\right)$ converges to a steady state. 
Next, for an oscillator $i$ of the coupled network, with $i \neq$ $1, k$, from (22) it holds that

$$
\frac{d R_{i}}{d t}=V_{R} \delta_{i}(t)-\left(\gamma_{R}+\kappa\right) R_{i}+\kappa \tilde{R}_{1}
$$

Since $\tilde{R}_{1}$ remains constant and the coupling parameter $\kappa$ is fixed, the term $\kappa \tilde{R}_{1}$ acts like a basal term for all components $R_{i}, i=2, \ldots, N$. This implies that systems $\left(A_{i}, R_{i}\right)$ become decoupled, and are described by the following equations:

$$
\begin{aligned}
& \frac{d A_{i}}{d t}=V_{A} s_{A, R}^{+}\left(A_{i}, R_{i} ; \theta_{0}, \theta_{2}\right)-\gamma_{A} A_{i}+r_{b a s} \\
& \frac{d R_{i}}{d t}=V_{R} s_{A}^{+}\left(A_{i} ; \theta_{1}\right)-\left(\gamma_{R}+\kappa\right) R_{i}+\kappa \tilde{R}_{1}
\end{aligned}
$$

This implies that each $i$-th system (36) is equivalent to system (18) with $\beta=\kappa, C=\tilde{R}_{1}$ and other parameters as in Fig. 3.

Since there are at least two oscillators in the network ( $k$ and 1) that converge to steady states, it holds that $\kappa>\hat{\kappa}$ (Theorem 2). From Lemma 5, it follows that either $\mu\left(a_{b a s}\right)<\tilde{R}_{1}$ or $\tilde{R}_{1}<\mu\left(a_{\max }\right)$. Recalling (21), we have that

$$
\begin{gathered}
\tilde{R}_{1}>\mu\left(a_{\text {max }}\right) \Rightarrow \beta_{1}\left(\tilde{R}_{1}\right)<\frac{\gamma_{R} \mu\left(a_{\text {bas }}\right)}{\mu\left(a_{\text {max }}\right)-\mu\left(a_{\text {bas }}\right)}<\hat{\kappa} \\
\tilde{R}_{1}<\mu\left(a_{\text {bas }}\right) \Rightarrow \beta_{2}\left(\tilde{R}_{1}\right)<\frac{V_{R}-\gamma_{R} \mu\left(a_{\text {max }}\right)}{\mu\left(a_{\text {max }}\right)-\mu\left(a_{\text {bas }}\right)}<\hat{\kappa}
\end{gathered}
$$

with the $\hat{\kappa}$ expression for $N$ sufficiently large.

For the range $\tilde{R}_{1} \in\left(\mu\left(a_{\text {bas }}\right), \mu\left(a_{\max }\right)\right)$, note that $\beta_{1}(C)$ is a decreasing function in the interval $\left(\mu\left(a_{b a s}\right),+\infty\right)$, with $\lim _{C \rightarrow \mu\left(a_{\text {bas }}\right)} \beta_{1}(C)=+\infty$ and, conversely, $\beta_{2}(C)$ is an increasing function in the interval $\left(0, \mu\left(a_{\text {max }}\right)\right.$ with $\lim _{C \rightarrow \mu\left(a_{\max }\right)} \beta_{2}(C)=+\infty$. Therefore, in the interval $C \in$ $\left(\mu\left(a_{\text {bas }}\right), \mu\left(a_{\text {max }}\right)\right)$, the two functions intersect at a single point $\hat{C}$ and it is easy to check that:

$$
\min \left\{\beta_{1}\left(\tilde{R}_{1}\right), \beta_{2}\left(\tilde{R}_{1}\right)\right\}<\beta_{1}(\hat{C})=\beta_{2}(\hat{C})
$$

with

$$
\beta_{1}(\hat{C})=\beta_{2}(\hat{C})=\frac{V_{R}-\gamma_{R}\left(\mu\left(a_{\text {bas }}\right)-\mu\left(a_{\text {max }}\right)\right)}{m u\left(a_{\text {bas }}\right)-\mu\left(a_{\text {max }}\right)} \equiv \hat{\kappa} .
$$

Thus, from (21), the $i$-th system (36) does not admit a periodic solution, meaning that all systems in the star-coupled network converge to a (locally) stable steady state.

\section{All-to-all topology}

An alternative configuration mimics the network of interconnections that might be found between the cells in a living tissue, where each cell communicate with all the others. This is a fully symmetrical network, since no oscillator can be distinguished from another. The corresponding $\Gamma_{R}+L$ matrix and its inverse are:

$$
\begin{aligned}
\Gamma_{R}+L & =\kappa\left(N I-\mathbf{1 1}^{\prime}\right)+\gamma_{R} I \\
\left(\Gamma_{R}+L\right)^{-1} & =\frac{1}{N \kappa+\gamma_{R}} I+\frac{\kappa}{\gamma_{R}\left(N \kappa+\gamma_{R}\right)} \mathbf{1 1},
\end{aligned}
$$

where $I$ is the $N \times N$ identity matrix, $\mathbf{1}$ is the vector with all entries equal to 1 and 11' is the matrix with all entries 1 .

As for the star topology, we can count the number of new steady states due to the coupling. Since all oscillators have an equal role, let $\left(D_{11}^{m} \times D_{00}^{N-m}\right)_{\text {prm }}$ denote any permutation of $m$ $D_{11}$ and $N-m D_{00}$ domains.

Theorem 4. Consider system (23) coupled in an all-to-all topology, with L defined as in (37). Assume that conditions (a)-(f) hold. Define:

$$
\begin{aligned}
& m_{\text {min }}=\left\lceil\frac{\gamma_{R}}{\kappa} \frac{N \kappa+\gamma_{R}}{V_{R}} \mu\left(a_{\text {bas }}\right)\right] \\
& m_{\text {max }}=\left\lfloor\frac{\gamma_{R}}{\kappa}\left(\frac{N \kappa+\gamma_{R}}{V_{R}} \mu\left(a_{\text {max }}\right)-1\right)\right\rfloor,
\end{aligned}
$$

If $m_{\min }<m_{\max }$, then system (23) has exactly $\sum_{m=m_{\min }}^{m_{\max }} N ! /(m !(N-m) !) \quad$ locally stable steady states corresponding to focal points $\phi_{C} \in D_{C}=\left(D_{11}^{m} \times D_{00}^{N-m}\right)_{\text {prm }}$. Furthermore, let

$$
\hat{\kappa}=\frac{1}{N}\left(\frac{V_{R}}{\mu\left(a_{\max }\right)-\mu\left(a_{\text {bas }}\right)}-\gamma_{R}\right)
$$

then none of the focal points corresponds to a steady state for any $\kappa \leq \hat{\kappa}$.

Proof. As in the proof of Theorem 2, we will provide conditions such that regular domains of the form $D_{C}=\left(D_{11}^{m} \times\right.$ $\left.D_{00}^{N-m}\right)_{\text {prm }}$ contain their own focal points. In this case, all oscillators play a similar role. By (24) and (37), the $\tilde{R}^{C}$ coordinates of the focal point of $D_{C}$ are given by

$$
\tilde{R}_{i}^{C}= \begin{cases}V_{R} \frac{\kappa}{\gamma_{R}(N \kappa+\gamma)} m, & \text { if } s h_{A, i}=0 \\ V_{R} \frac{1}{N \kappa+\gamma_{R}}+V_{R} \frac{\kappa}{\gamma_{R}\left(N \kappa+\gamma_{R}\right)} m, & \text { if } s h_{A, i}=1 .\end{cases}
$$

By Lemma 5, the conditions to guarantee that $\phi_{C} \in D_{C}$

$$
\begin{aligned}
& V_{R} \frac{\kappa}{\gamma_{R}\left(N \kappa+\gamma_{R}\right)} m>\mu\left(a_{\text {bas }}\right) \\
& V_{R} \frac{1}{N \kappa+\gamma_{R}}+V_{R} \frac{\kappa}{\gamma_{R}\left(N \kappa+\gamma_{R}\right)} m<\mu\left(a_{\text {max }}\right),
\end{aligned}
$$

immediately yield the values (39). Since the interconnection scheme is fully symmetric, the maximal number of new steady states equals all the possible combinations of the form $D_{C}$, that is $N ! /(m !(N-m) !)$, for each $m_{\min } \leq m \leq m_{\max }$.

The value $\hat{\kappa}$ is the highest value that implies $m_{\min }>m_{\max }$ in (39). It follows by solving the inequality with respect to $\kappa$ :

$$
\gamma_{R}\left(\gamma_{R}+N \kappa\right)\left(\mu\left(a_{\max }\right)-\mu\left(a_{b a s}\right)\right)-V_{R} \gamma_{R}<0
$$




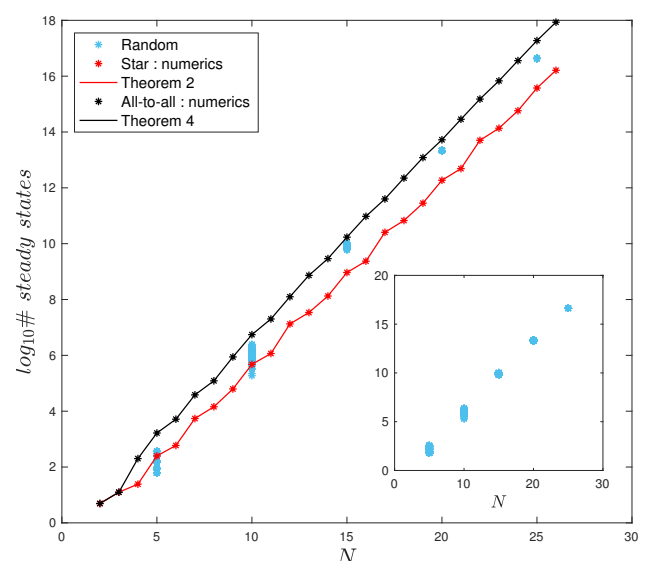

Figure 6 . The number of new steady states (log scale) generated by each topology.

A fundamental difference arises between the star and the all-to-all topology: the upper bound on the diffusion constant decreases to zero with $N$ in the all-to-all topology, while it remains constant in the star topology (Fig. 7). For large $N$, we can expect to avoid new steady states in the star topology by assigning a small but finite and reasonable diffusion constant (on the order of the degradation constants); however, steady states will appear for sufficiently large $N$ in all-to-all topology, possibly contributing to disrupt synchronization of the oscillators to the periodic orbit. In any case, the periodic orbit is preserved: in the last section we give the basin of attraction for the periodic solution. Therefore, considering clusters of cells connected in all-to-all topology, for a reasonable number of cells in each cluster the oscillations are preserved regardless the large number of the steady states generated by the coupling, see Fig.8.

\section{E. Random topology}

Star and all-to-all topologies are standard network architectures in graph theory with numerous applications. When it comes to biology, in particular to intercellular coupling, it becomes important to explore a larger class of interconnection structures.

In the work of Hafner, Koeppl, and Gonze (2012), a comprehensive mathematical model of the circadian clock is used to simulate the cells inside the SCN with different types of interconnections. The results of this study suggest that cells with problematic oscillatory behaviour synchronize more efficiently in a random topology than in other topologies tested. Motivated by this result, we introduce a random topology for the network (23), with degree of connectivity $d$. The cell $i$ is randomly bidirectionally connected to cell $j$, with $i, j=1, \ldots, N, i \neq j$, and each cell (node) has exactly $d$ connections (edges). We will denote this bidirectional connection as : $i \leftrightarrow j$. The elements of the Laplacian matrix $L$ are defined

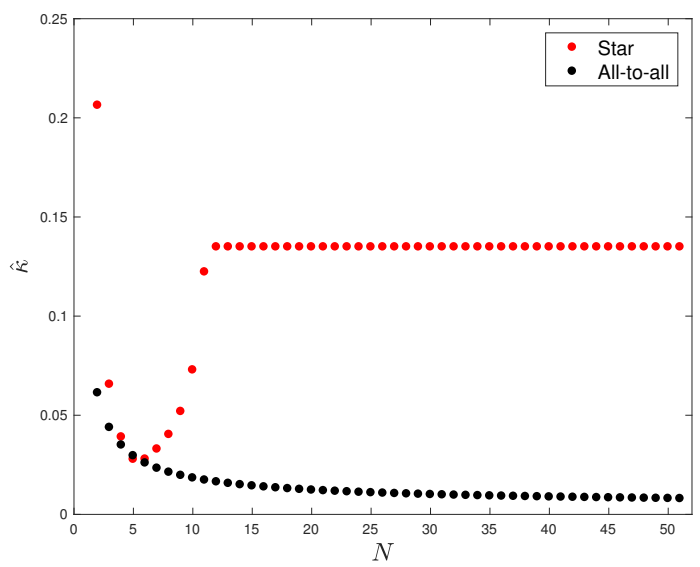

Figure 7. The upper bound $\hat{\kappa}$ on the diffusion constant that guarantees no new steady states for $\kappa<\hat{\kappa}$.

as

$$
l_{k j}=\left\{\begin{array}{l}
\kappa(N-1-d), \quad j=k \\
-\kappa, \quad \text { if } j \leftrightarrow k, j \neq k,
\end{array}\right.
$$

The numerical results for the total number of new locally stable steady states in random connected networks of $N$ oscillators, with degree of connectivity fixed for each network at $25 \%-50 \%$ of $N$, are illustrated in Fig. 6, along with the corresponding numbers for the two other topologies.

The comparison of the total number of steady states for the three topologies, shows that random connections generate more steady states than star topology but less than all-to-all. This is an intuitively reasonable result since, for the average degree of connectivity $\delta$, for the three types of connections, we have that $\delta_{\text {star }}<\delta_{\text {random }}<\delta_{\text {all-to-all }}$.

\section{F. Initial conditions to guarantee convergence to the periodic solution}

The generation of new stable steady states due to the diffusive coupling may be a disrupting factor in a network where the goal is synchronization towards the biological rhythm present in each single cell. Thus, in addition to the lower bound on the coupling constant, which prevents the generation of new stable steady states, it is also crucial to define initial conditions that guarantee convergence to the periodic orbit. Due to the specific form of the domains of system (9), and consequently of system (22) (that are defined by the quadratic curve $\mu(A)$ ), the explicit definition of the basins of attraction for the limit cycle and the locally stable steady states is not straightforward. Instead, numerical simulations were performed to identify the basins of attraction for the two types of attractors in three regions (zones) of the phase space, as defined in Table III. Table IV shows the percentage of initial conditions that converge to the steady states in each zone.

To facilitate the characterization of our results, first we define the line $l_{\theta}(A)=c A+b$, that passes through the point $\left(\sqrt{\theta_{0}}, 0\right)$ and intersects the R-nullcline $A=\sqrt{\theta_{1}}$ and the $R$ 
Table III. Phase space of system (22) partitioned in three regions to test convergence of initial conditions. Parameters as in Fig. 3, $l_{\theta}(A)=c A+b, c=0.8482, b=-0.7082$.

\begin{tabular}{c||c} 
Zones & \\
\hline 1 & $\left\{R_{i} \in\left[0, V_{R} / \gamma_{R}\right), A_{i} \leq l_{\theta}^{-1}\left(R_{i}\right)\right\}$ \\
\hline 2 & $\left\{R_{i} \in\left[0, V_{R} / \gamma_{R}\right), A_{i}>l_{\theta}^{-1}\left(R_{i}\right) \cap\left(\sqrt{\theta_{1}}+\varepsilon, \mu^{-1}\left(R_{i}\right)\right)\right\}$ \\
\hline 3 & $A_{i} \in\left(\sqrt{\theta_{1}}+\varepsilon, \mu^{-1}\left(V_{R} / \gamma_{R}\right)\right), R_{i} \in\left[0, \mu\left(A_{i}\right)\right)$ \\
\hline
\end{tabular}

Table IV. Percentage of the initial conditions that lead the trajectories to the steady states, $N=10, \kappa=0.25$, and the initial states for each system to slightly vary as: $\left(A_{i}, R_{i}\right)=\alpha\left(A_{1}, R_{1}\right)$, with $\left(A_{1}, R_{1}\right) \in$ zone $j, \alpha \in[0.8,1.2], i=2, \ldots N$ and $j=1,2,3$.

\begin{tabular}{c||c|c|c} 
Topology & Star & All-to-all & Random \\
\hline Zone 1 & $0 \%$ & $0 \%$ & $0 \%$ \\
\hline Zone 2 & $15.2 \%$ & $43.6 \%$ & $27-39 \%$ \\
\hline Zone 3 & $4.7 \%$ & $25.8 \%$ & $15-21.7 \%$ \\
\hline
\end{tabular}

threshold-line $R=V_{R} / \gamma_{R}$, with $c=V_{R} / \gamma_{R}\left(\sqrt{\theta_{1}}-\sqrt{\theta_{0}}\right)$ and $b=-V_{R} \sqrt{\theta_{0}} / \gamma_{R}\left(\sqrt{\theta_{1}}-\sqrt{\theta_{0}}\right)$. This line is used to define the zones of Table III.

The results of Table IV imply that trajectories starting in zone 1 have the highest probability to oscillate, with $100 \%$ of the initial conditions tested leading to the periodic orbit. We note that the other two zones both intersect the basins of attraction of more than one attractor. Initial conditions in the region

$$
\begin{array}{r}
C=\left\{(A, R) \in \mathbb{R}_{\geq 0}^{2 N}\right\}, R_{i} \in\left[0, V_{R} / \gamma_{R}\right), A_{i} \leq l_{\theta}^{-1}\left(R_{i}\right), \\
i=1, \ldots N
\end{array}
$$

lead trajectories of system (22) to the periodic solution, for

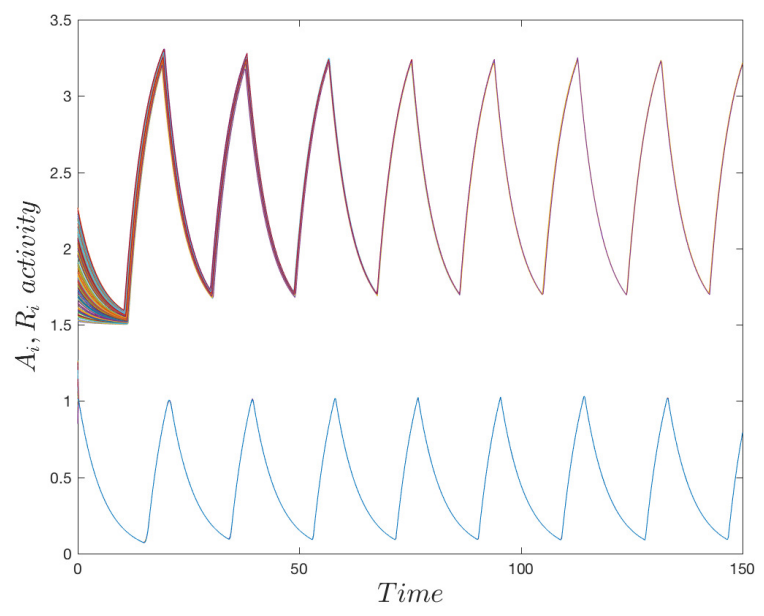

Figure 8. Activity in time of $N=200$ coupled two-gene oscillators of the form (9) in all-to-all topology, initial conditions in zone 1, $\kappa=0.25$ and other parameters as in Fig. 3. Trajectories converge to the periodic solution.

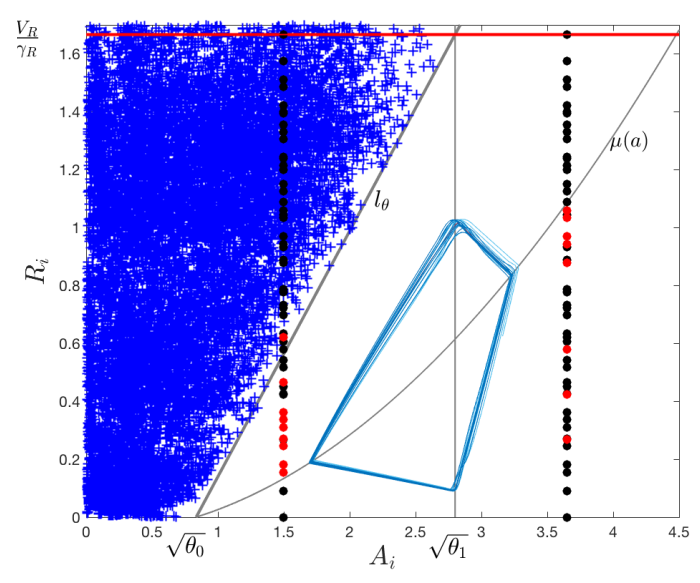

Figure 9. For all initial conditions tested in zone 1 (blue + symbols), trajectories converge to the periodic orbit. Here are shown the numerical tests for star topology with $N=10$.

the three topologies tested. Indicatively for star topology, initial conditions in zone 1 are depicted in Fig. 9; trajectories starting from the marked superimposed points $\left(A_{i}, R_{i}\right)$ converge to the periodic orbit.

\section{DISCUSSION}

An improved synthetic oscillator with PWA analysis In this work we study an improved model of a synthetic biology oscillator using a piecewise affine approximation and prove existence and uniqueness of periodic solution for this PWA system, in a suitable region of parameters. This qualitative characterization of the system allows us to better understand the influence of each parameter on the dynamical behaviour, crucial factors to help guide system design and synthetic implementation. The two most important steps for the improvement of the system design are: first, separate the activation thresholds. The activator should act much faster on itself than on the repressor $\left(\theta_{0}<\theta_{1}\right)$. Second, the maximal rate for the activator synthesis $\left(V_{A}+r_{\text {bas }}\right) / \gamma_{A}$ should be smaller than the maximal repressor modulating factor $\sqrt{1+V_{R} /\left(\gamma_{R} \theta_{2}\right)}$.

Interconnection of identical oscillators generates new $d y$ namics A network of synthetic oscillators was next constructed by connecting $N$ identical elements through diffusive coupling, organized according to different interconnection structures: one-to-all or star, all-to-all and random. All three forms of interconnection preserve the periodic orbit but acquire additional attractors: new locally stable steady states. We give the exact total number of the new stable states, which increases with the number $N$ of the systems in the network. In addition, we provide a lower bound on the diffusion constant that prevents the generation of new steady states due to the coupling. We also identify a region of the state space which prevents convergence to any of the steady states, for the three forms of interconnections applied. Numerical simulations show that, in the case of convergence to the periodic 
orbit, the $N$ systems synchronize both in period and in phase, even for a small value of the coupling constant. An interesting question to be addressed in future work is whether the coupled systems still synchronize if the individual systems are not identical but have different parameters and periods.

Network topologies and robustness of oscillations The three interconnection topologies lead to similar qualitative dynamical behaviours and raise the problem of the robustness of the periodic behavior, when the goal is, for instance, the synchronization of a cluster of cells to the same periodic rhythm. The analysis of the new steady states and their basins of attraction indicates that, for a large amount of initial conditions, the network elements will not synchronize to a similar asymptotic behaviour. Star topology has fewer new steady states and a finite lower bound on the diffusion constant that prevents locally stable steady states and guarantees the existence of periodic oscillations. Moreover, this lower bound has physiologically reasonable values and is independent of the number of cells in the network. In constrast, the all-to-all topology creates locally stable steady states for any value of diffusion constant, for sufficiently large $N$. This analysis indicates that a star interconnection, resembling a central synchronizer with peripheral clocks, has the capacity to more robustly maintain a synchronized oscillatory behaviour, while in an all-to-all network, representing identical cells in a homogeneous tissue, it will be difficult to synchronize to a periodic orbit as the number of cells increases. In random interconnections, reminiscent of a network of circadian oscillators inside the SCN, the total number of locally stable states lies in between the corresponding numbers of the two other topologies, making this type of connection more robust to oscillations than all-to-all topology.

Conclusion Our results show that the architecture of interconnections induces new dynamics in a network of identical oscillators, creating new steady states and interfering with synchronization properties, which can be undesirable in synthetic biology applications. Our analysis provides useful guiding lines for choosing network design and implementation showing that, by carefully adjusting the coupling strength or tuning initial conditions, it is still possible to synchronize the network of $N$ biological oscillators, in all architectures. However, a star-shaped network has the potential to more robustly maintain oscillators synchronized.

\section{ACKNOWLEDGMENTS}

We gratefully thank Luca Scardovi for many interesting and useful discussions on the coupling and synchronization of oscillators, during his extended visit to Inria. The authors were partly supported by the French national agency for research through project ICycle ANR-16-CE33-0016-01.

\section{DATA AVAILABILITY}

The data that supports the findings of this study are available within the article.

\section{REFERENCES}

Balsalobre, A., Damiola, F., and Schibler, U., "A serum shock induces circadian gene expression in mammalian tissue culture cells," Cell 93, 929 937 (1998).

Barbier, I., Perez-Carrasco, R., and Schaerli, Y., "Controlling spatiotemporal pattern formation in a concentration gradient with a synthetic toggle switch.” Mol Syst Biol 16, e9361 (2020).

Casey, R., Jong, H. d., and Gouzé, J.-L., "Piecewise-linear models of genetic regulatory networks: Equilibria and their stability," Journal of Mathematical Biology 52, 27-56 (2006).

Chaves, M., Scardovi, L., and Firippi, E., "Coupling and synchronization of piecewise linear genetic regulatory systems," in IEEE Conference on Decision and Control (Nice, France, 2019).

del Vecchio, D., Dy, A., and Qian, Y., "Control theory meets synthetic biology,” J. R. Soc. Interface 13, 20160380 (2016).

Edwards, R. and Gill, P., "On synchronization and cross-talk in parallel networks,” Dyn. Contin.Discrete Impuls. Syst. Ser. B Appl. Algorithms 10, 287-300 (2003).

Elowitz, M. B. and Leibler, S., "A synthetic oscillatory network of transcriptional regulators," Nature 403, 335-338 (2000).

Filippov, A. F., Differential equations with discontinuous right-hand side, Mathematics and its Applications, Vol. 19 (Kluwer Academic Publishers, Dordrecht, Boston, London, 1960) pp. 99-128.

Fiore, G., Matyjaszkiewicz, A., Annunziata, F., Grierson, C., Savery, N. J., Marucci, L., and di Bernardo, M., "In-silico analysis and implementation of a multicellular feedback control strategy in a synthetic bacterial consortium," ACS Synth. Biol. 6, 507-517 (2017).

Firippi, E. and Chaves, M., "Period -control in a coupled system of two genetic oscillators for synthetic biology," in FOSBE 2019 - 8th IFAC Conference on Foundations of Systems Biology in Engineering (Valencia, Spain, 2019).

Gardner, T., Cantor, C., and Collins, J., "Construction of a genetic toggle switch in escherichia coli," Nature 403, 339-42 (2000).

Glass, L. and Kauffman, S. A., "The logical analysis of continuous, non-linear biochemical control networks," Journal of Theoretical Biology 39, 103 129 (1973).

Hafner, M., Koeppl, H., and Gonze, D., "Effect of network architecture on synchronization and entrainment properties of the circadian oscillations in the suprachiasmatic nucleus," PLOS Computational Biology 8, 1-16 (2012).

Nicks, R., Chambon, L., and Coombes, S., "Clusters in nonsmooth oscillator networks,” Phys. Rev. E 97, 032213 (2018).

Perez-Carrasco, R., Barnes, C. P., Schaerli, Y., Isalan, M., Briscoe, J., and Page, K. M., "Combining a toggle switch and a repressilator within the ac-dc circuit generates distinct dynamical behaviors," Cell Systems, Cell Systems 6, 521-530.e3 (2018).

Purcell, O., Savery, N. J., Grierson, C. S., and di Bernardo, M., “A comparative analysis of synthetic genetic oscillators.” J R Soc Interface 7, 15031524 (2010).

Ralph, M., Foster, R., Davis, F., and Menaker, M., “Transplanted suprachiasmatic nucleus determines circadian period," Science 247, 975-978 (1990), https://science.sciencemag.org/content/247/4945/975.full.pdf.

Silver, R., LeSauter, J., Tresco, P. A., and Lehman, M. N., "A diffusible coupling signal from the transplanted suprachiasmatic nucleus controlling circadian locomotor rhythms," Nature 382, 810-813 (1996).

Smolen, P., Baxter, D. A., and Byrne, J. H., "Frequency selectivity, multistability, and oscillations emerge from models of genetic regulatory systems," American Journal of Physiology - Cell Physiology 274, C531C542 (1998), http://ajpcell.physiology.org/content/274/2/C531.full.pdf.

Stricker, J., Cookson, S., Bennett, M. R., Mather, W. H., Tsimring, L. S., and Hasty, J., "A fast, robust and tunable synthetic gene oscillator." Nature 456, 516-519 (2008).

Yamazaki, S., Numano, R., Abe, M., Hida, A., Takahashi, R.-i., Ueda, M., Block, G. D., Sakaki, Y., Menaker, M., and Tei, H., "Resetting central and peripheral circadian oscillators in transgenic rats," Science 288, 682-685 (2000), https://science.sciencemag.org/content/288/5466/682.full.pdf. 